PALABRAS CLAVE

Industria automotriz

Comercio internacional

Mercosur

Acuerdos económicos

Exportaciones

Argentina

Brasi

Estadísticas comerciales

Valeria Arza

Investigadora del Consejo Nacional de Investigaciones Científicas y Técnicas (CONICET)

Centro de Investigaciones para la

Transformación (CENIT)

œ varza@fund-cenit.org.ar
REVISTA CEPAL 103 A BRIL 2011

\section{El Mercosur como plataforma de exportación para la industria automotriz}

\author{
Valeria Arza
}

$\mathrm{L}$

industria automotriz mundial está dominada por unas pocas empresas multinacionales que diseñan las estrategias a nivel global y regional. Si las estrategias regionales prevalecieran sobre las globales, el Mercado Común del Sur (Mercosur) podría transformarse en una plataforma de exportación competitiva. En este artículo se examina hasta qué punto los acuerdos de comercio para la industria automotriz establecidos en el Mercosur promovieron que la región se transformara en una plataforma de exportación para el resto del mundo. Se emplean datos de comercio bilateral del período 1991-2005 y modelos de gravedad para evaluar la creación de comercio y la diversificación del mercado de exportación de la industria automotriz. Los resultados muestran que hasta 2005 los acuerdos del Mercosur no convirtieron a la región en una plataforma exportadora para los mercados exteriores, aunque contribuyeron a la creación de comercio intrarregional. 


\section{I}

\section{Introducción}

La producción automotriz se halla bajo el dominio de unas pocas empresas multinacionales. En 2005, las cinco principales (General Motors, DaimlerChrysler, Toyota, Ford y Volkswagen) representaban el 65\% de la producción total. Las subsidiarias de algunas de estas empresas comenzaron su producción en la Argentina y el Brasil en la década de 1950, motivadas principalmente por el incremento de los mercados internos de estos países, que se encontraban muy protegidos como suele ocurrir en general con el mercado automotor de los países productores.

Sin embargo, el desarrollo de nuevas tendencias en la industria en la década de 1990 debilitó el papel que tenían los mercados internos, así como los incentivos para un mayor incremento de la capacidad de producción de las subsidiarias de empresas multinacionales. Estas nuevas tendencias se orientaron a acrecentar la competitividad internacional mediante la internacionalización de la producción, estrategia considerada como una forma eficaz de reducir costos y aumentar al mismo tiempo la variedad de productos en todo el mundo. En este contexto, las políticas proteccionistas dejaban de ser el mejor incentivo para crear una industria automotriz internacionalmente competitiva, dado que las estrategias empresariales contenían fuertes componentes globales que perpetúan la necesidad de un intenso comercio internacional, tanto de vehículos como de partes o piezas, dentro de la corporación y con proveedores del exterior.

La amplia adopción de estrategias globales por parte de las empresas multinacionales en el sector automotor debería haber creado un "automóvil global" o, en otras palabras, un automóvil producido a nivel global para mercados globales. Sin embargo, la evidencia parece indicar que las terminales automotrices tienden a concentrar sus ventas en las regiones donde se ubican sus casas matrices y que localizan las subsidiarias estratégicamente para capturar los mercados de las proximidades de los lugares de producción. Estas estrategias regionales se implementan de forma simultánea y, de alguna manera, complementan las estrategias globales.

En la sección II se examinan con más detalle estas estrategias globales y regionales, lo que corrobora las conclusiones de muchos autores (Freyssenet y Lung, 2000; Humphrey y Memedovic, 2003, Rugman y Collinson,
2004) según los cuales las estrategias regionales de la industria automotriz son más eficientes y rentables que las estrategias nacionales o globales. Esto ofrece perspectivas alentadoras para el Mercosur, ya que podría transformarse en una plataforma de producción y exportación de una industria internacionalmente competitiva.

La integración entre los miembros del Mercosur (la Argentina, el Brasil, el Paraguay y el Uruguay) aún no se ha alcanzado plenamente en lo que atañe a la industria automotriz. En su lugar, existe una serie de acuerdos bilaterales entre socios. Los más importantes son los firmados entre la Argentina y el Brasil, dado que ambos representan casi el 100\% de la producción de automóviles en el Mercosur. Estos países firmaron un acuerdo especial de comercio para el sector automotor a fines de 1994. El proceso de integración se intensificó aún más a consecuencia de un segundo acuerdo firmado en el año 2000. El propósito de este artículo es conocer en qué medida los acuerdos de 1994 y 2000 se tradujeron en la creación de comercio genuino y también si han facilitado la diversificación del mercado de exportación.

En este artículo se procura dar respuesta a las siguientes preguntas de investigación: ¿Hay evidencia de creación de comercio después de 1994 y con posterioridad al año 2000? ¿Existen patrones de diversificación del mercado de exportación después de esas fechas? ¿Estos patrones son similares en los casos de la Argentina y el Brasil? ¿La diversificación surge a expensas del comercio en el interior del bloque? ${ }^{1}$

El enfoque metodológico se apoya en el concepto de competitividad revelada. Se analizará en qué medida aumentó en estos países la intensidad del comercio "intrabloque", la diversificación de las exportaciones a los mercados "extrabloque", o ambas, en los años posteriores a la celebración de los acuerdos. Se fusionaron varias bases de datos para estimar los modelos de gravedad sectorial y se elaboró un panel no balanceado de 59.165 flujos bilaterales de comercio del sector automotor (CIIU Rev. $2 \mathrm{~N}^{\circ} 341$ ) entre todos los países del mundo durante el período 1991-2005.

\footnotetext{
${ }^{1}$ El término "bloque" usado en este artículo hace referencia a la asociación entre ambos países.
} 
En la sección II, como se indicó, se analizan las tendencias globales y regionales de la industria; en la sección III se contextualiza el estudio mediante la descripción de los principales rasgos del marco regulatorio y se presentan también las principales estadísticas de producción y comercio de la industria automotriz en la
Argentina y el Brasil; en la sección IV se presentan las preguntas de investigación y las hipótesis. En la sección V se describe la metodología para la prueba de las hipótesis; en la sección VI se examinan los resultados empíricos y, por último, en la sección VII se presentan las conclusiones.

\section{II}

\section{Tendencias internacionales}

\section{de la industria automotriz ${ }^{2}$}

De 1961 a 2005 la producción global de automóviles se incrementó un 337\%, lo que significa una tasa de crecimiento anual acumulado del $3 \%$. Este proceso de expansión ha ido acompañado de una concentración cada vez mayor del mercado automotor global que, como se dijo, actualmente se encuentra dominado por unas pocas e importantes empresas multinacionales.

A pesar de la concentración del mercado, los Estados Unidos fueron perdiendo peso en la distribución de la producción mundial. Este país registraba el $44 \%$ del total de vehículos producidos en 1961, pero hacia 2005 este porcentaje se redujo al $18 \%$. En tanto, la participación global de otras regiones, como Asia (y China en particular), aumentó notablemente. Esta relocalización de la producción podría explicarse por el surgimiento e intensificación de las estrategias corporativas globales y regionales.

En la década de 1990 aparecieron tendencias globales en la industria dirigidas principalmente a acrecentar la competitividad mediante la reducción de los costos y el incremento de la variedad de productos. Estas tendencias, que condujeron a la reorganización de la cadena de valor y a la internacionalización de la producción, se conocen como "estandarización” (commonalisation), "modularización" (modularisation) y "globalización de proveedores" (global sourcing). ${ }^{3}$

La “estandarización” consiste en el uso común en todo el mundo de plataformas y otros componentes mecánicos para concentrar la mayoría de las actividades

\footnotetext{
${ }^{2}$ Esta sección se basa en gran parte en Arza y López (2008a).

${ }^{3}$ Más detalles sobre estas estrategias globales pueden obtenerse en Humphrey, Lecler y Salerno (2000).
}

de diseño en unos pocos lugares. ${ }^{4}$ Esto ofrece nuevas posibilidades de incrementar la escala (sobre todo en el diseño y el desarrollo) y las economías de alcance, ya que — con pocas modificaciones — pueden producirse diferentes modelos y versiones en las mismas plataformas. Las actividades de diseño se localizan habitualmente en los países centrales, de modo que los países en desarrollo adoptan por lo general la estrategia de seguir el diseño (follow design), lo que implica que rara vez intervendrán en el diseño de sus propios modelos. En su lugar, adoptarán modelos diseñados de manera centralizada por la empresa. ${ }^{5}$ No obstante, a partir de las estrategias regionales de las empresas se crean algunas ventanas de oportunidad para las actividades de diseño realizadas por subsidiarias en países en desarrollo, sobre todo cuando se promueven mediante políticas regionales (véase el caso de Fiat-Brasil en Ciravegna, 2003).

La "modularización" supone cambios en la arquitectura de producción de automóviles, que pasa del ensamble de partes al ensamble de subsistemas. La producción de estos subsistemas puede externalizarse y algunos proveedores especiales (a veces denominados megaproveedores) pueden producir un módulo individual para un subsistema completo (paneles de instrumentos, asientos, cajas de cambio o puertas, entre otros). Por lo tanto, la modularización también involucra la existencia de mayores responsabilidades para los megaproveedores

\footnotetext{
${ }^{4}$ El concepto de plataforma incluye el chasis, la suspensión, la transmisión y el espacio donde se inserta el motor, entre otros elementos (Bastos Tigre y otros, 1999).

${ }^{5}$ Esto se diferencia claramente de la lógica tecnoproductiva de los años sesenta y setenta, cuando los distintos modelos se producían y vendían en los mercados nacionales y regionales con importantes innovaciones adaptativas de diversas subsidiarias localizadas alrededor del mundo (Cimoli y Katz, 2001).
} 
$\mathrm{y}$, en consecuencia, las terminales automotrices han establecido de manera creciente relaciones simbióticas con ellos. Por ejemplo, hoy en día es común observar que los proveedores y las terminales automotrices participan simultáneamente en actividades de ingeniería (cooperación en la generación de nuevos productos, procesos o ambos). La dependencia cada vez mayor respecto de los proveedores promovió relaciones de largo plazo con una menor cantidad de ellos, en lugar de alentar la competencia entre un gran número de potenciales proveedores, que era la estrategia aplicada en décadas anteriores. Asimismo, a medida que los proveedores tienen mayor participación en las actividades de producción, las terminales automotrices se especializan cada vez más en actividades de diseño.

Las tendencias de "estandarización" y "modularización" conducen en cierta medida a la tercera tendencia: la "globalización de proveedores". Dado que se emplean componentes comunes para producir diferentes modelos y que los proveedores se transforman en actores clave en la producción de automóviles, las terminales automotrices prefieren usualmente comprar a los mismos proveedores, independientemente del lugar donde se lleve a cabo la producción. Esto plantea a los proveedores (sobre todo a los megaproveedores y a otros proveedores en el primer anillo) la necesidad de globalizar su actividad (esta tendencia no alcanza a los proveedores de componentes básicos del segundo y tercer anillo). ${ }^{6}$ Asimismo, dadas las tecnologías de producción y demanda sincronizadas (just-in-time), los proveedores globales a veces también necesitan seguir a las terminales automotrices a sus lugares de producción, estrategia que en la jerga se denomina "seguir abasteciendo" (follow sourcing). De todas maneras, esta estrategia se ve limitada cuando se requieren grandes economías de escala para lograr una producción eficiente.

En general, estas tendencias globales deberían conducir a las terminales automotrices a producir globalmente para la venta en todo el mundo. No obstante, la evidencia empírica parece indicar que: i) las empresas multinacionales concentran sus actividades de

\footnotetext{
${ }^{6}$ Los anillos hacen referencia a diferentes grupos de fabricantes de componentes y partes de vehículos ordenados de acuerdo con la sofisticación de su producción y el tipo de relación que establecen con la terminal automotriz. En el primer anillo se encuentran los fabricantes de partes con procesos de ingeniería y diseño incorporados, muchas veces desarrollados en forma modular. En el segundo anillo se encuentran los fabricantes de componentes que también abastecen a los del primer anillo. Finalmente, en el tercer anillo se hallan los fabricantes de componentes estandarizados que son insumos de la industria automotriz, pero también de otras industrias.
}

producción en la región donde se ubica su casa matriz y ii) las subsidiarias de las empresas multinacionales tienden a localizarse estratégicamente para proveer a los mercados regionales en las cercanías de sus lugares de producción.

En el gráfico 1 se agrupan en cuatro regiones las actividades de producción de las empresas multinacionales: Asia, América del Norte, Europa y otras. Como puede verse, ninguna de ellas es una verdadera empresa global si a esta se la define como aquella que efectúa por lo menos el $20 \%$ de su producción en cada una de las tres principales regiones de producción: Asia sigue siendo la plataforma más importante para la producción de Toyota (64\%) y Europa es la región donde las empresas realizan la mayor producción de las marcas PSA Peugeot Citroën (83\%), Renault (83\%), Volkswagen (Vw) (71\%) y Fiat (65\%). General Motors Company (GM) (56\%), DaimlerChrysler (DC) (54\%) y Ford (49\%) producen sobre todo en América del Norte. ${ }^{7}$

Esta evidencia, que enfatiza la relevancia de lo regional sobre lo puramente global, coincide con un debate que se plantea en la literatura especializada. De hecho, en varios estudios se argumenta que en las empresas globales predominan las estrategias regionales más que las globales. Se dice que las estrategias regionales son más rentables, sobre todo porque explotan mejor y al mismo tiempo las economías de escala y las economías de alcance (Rugman y Hodgetts, 2001).

Rugman y Collinson (2004) plantearon varias razones en favor del argumento de que es más probable que la industria automotriz intente localizar la producción en los mercados regionales, en vez de transformarse en una verdadera industria global. En primer lugar, la escala eficiente se logra habitualmente a nivel regional (Schlie y Yip, 2000), sobre todo desde que los acuerdos regionales de comercio se ampliaron y difundieron (Humphrey y Memedovic, 2003); en segundo lugar, la demanda a menudo se estratifica a nivel regional debido a patrones comunes culturales, ambientales y relativos a la reglamentación sobre seguridad y uso de combustibles, entre otros. Asimismo, las terminales automotrices también prefieren que sus socios en la cadena de valor operen

\footnotetext{
${ }^{7}$ Respaldan esta evidencia las conclusiones de Rugman y Collinson (2004), que analizaron datos de 2001 para todo el complejo automotor. Estos autores hallaron que ninguna de las 29 empresas automotrices (incluidas las terminales automotrices y los fabricantes de partes) entre las 500 más grandes del mundo podían denominarse "empresa global", es decir, una empresa en cuyo mercado se incluye por lo menos una porción del 20\% de cada una de las regiones que conforman la tríada (América del Norte, Asia y Europa).
} 
GRÁFICO 1

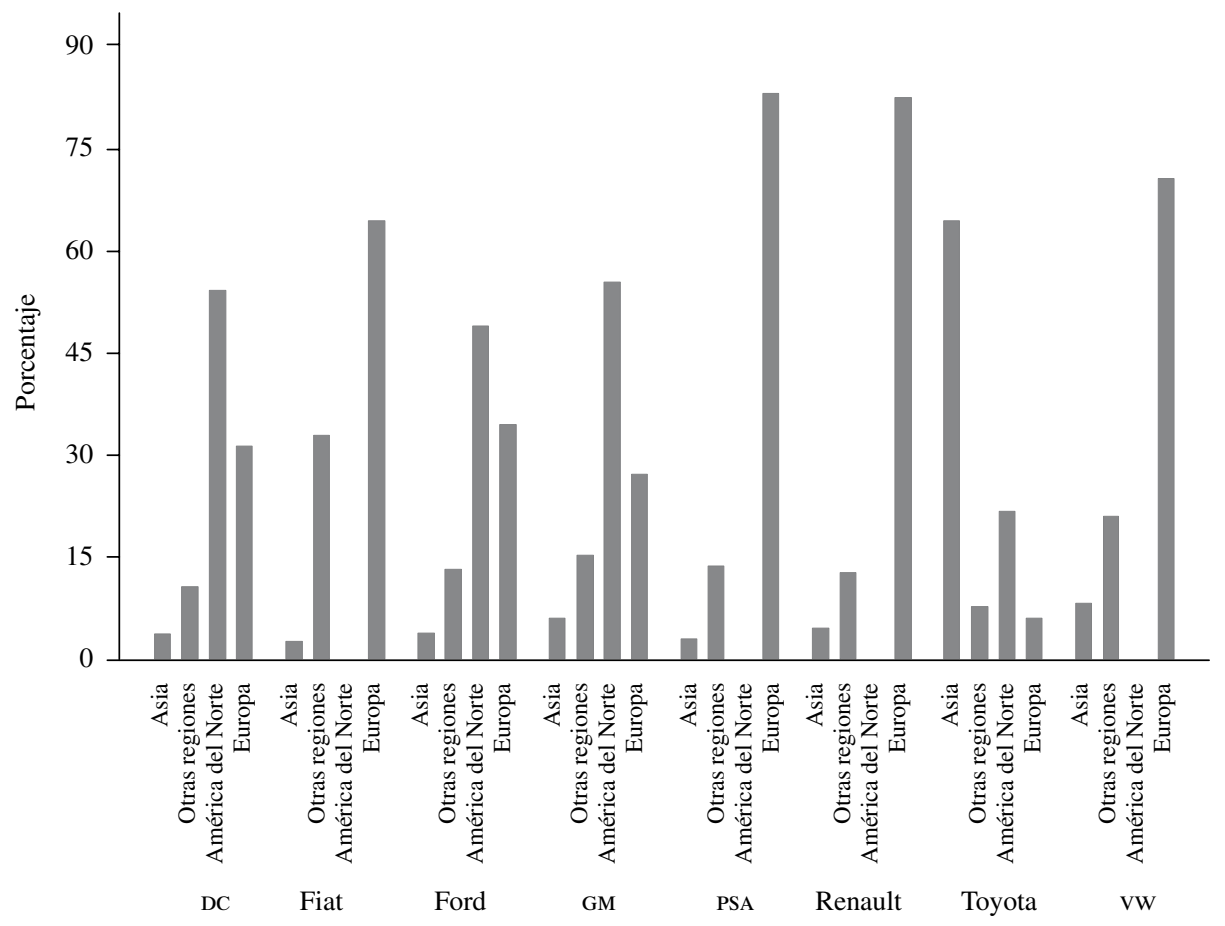

Fuente: elaboración propia sobre la base de datos de la Organización Internacional de Fabricantes de Automóviles.

en la misma región en que producen. ${ }^{8}$ Esto se debe a que una red bien establecida de distribución, servicios financieros y servicios de posventa en la región aumenta la rentabilidad de las terminales automotrices.

¿En qué medida estos cambios observados en la industria automotriz global repercuten en la producción de los países en desarrollo?

Un punto fundamental que es necesario considerar es que, a diferencia de lo ocurrido en el pasado, las políticas proteccionistas a nivel nacional ya no serán un factor de atracción per se para la inversión en este sector y podrían incluso transformarse en un factor negativo. Esto es así porque, como se señaló anteriormente, la actual lógica de producción en este sector tiene importantes componentes globales y regionales, lo que supone la necesidad de un comercio fluido de automóviles y piezas entre subsidiarias de las principales corporaciones multinacionales localizadas alrededor del mundo y entre

${ }^{8}$ Esta situación estaría indicando que existe un límite para la globalización de los proveedores. ellas y sus proveedores internacionales. Sin embargo, la prevalencia de estrategias regionales a nivel corporativo ofrece ventanas de oportunidad para una política comercial basada en acuerdos regionales.

Varios de estos acuerdos se han difundido en todo el mundo: el Mercosur es un caso interesante porque, junto con la Asociación de Naciones del Asia Sudoriental (ASEAN), es la única agrupación que solo comprende países en desarrollo. Si bien aún no se ha logrado un acuerdo pleno sobre la industria automotriz entre los países del Mercosur (la Argentina, el Brasil, el Paraguay y el Uruguay), punto que se analiza más adelante, en este artículo se intenta medir el efecto del acuerdo entre sus principales socios (la Argentina y el Brasil) en cuanto a la creación de comercio y la diversificación del mercado de exportaciones.

En la sección siguiente se describen los cambios en la regulación en lo que respecta a los acuerdos comerciales para el sector automotor entre estos países desde la década de 1990 y también se analiza la evolución de la producción y el comercio de la industria automotriz en la Argentina y el Brasil en el mismo período. 


\section{III}

\section{La industria automotriz en la Argentina y el Brasil}

El Mercosur es un espacio importante para las ventas y la producción mundial de la industria automotriz. En 2006 este mercado común de países se ubicaba en el séptimo lugar de la jerarquía internacional de países productores de vehículos (produjo 3 millones de vehículos), detrás del Japón (11,5 millones), los Estados Unidos (11,3 millones), China (7,2 millones), Alemania (5,8 millones), la República de Corea (3,8 millones) y Francia (3,2 millones de vehículos). Por debajo de los países del Mercosur se ubicaron: España (2,8 millones de vehículos), el Canadá (2,6 millones), México, la India y los países de la ASEAN ( 2 millones de vehículos cada uno de ellos). ${ }^{9}$ En 2006 se registraron 2,4 millones de vehículos nuevos en el Mercosur, cifra que ubica a la región en el octavo puesto de la jerarquía internacional. Además, América Latina ha tenido una larga trayectoria de producción en esta industria, que se inició a fines de la década de 1950. En muchos casos, las subsidiarias en el Mercosur fueron pioneras en las estrategias de internacionalización de las grandes empresas.

En esta sección se analiza en primer lugar la evolución de las normas específicas relativas a la integración del Mercosur en lo referido a la industria automotriz, desde 1994 hasta 2006. Se muestran estadísticas descriptivas con el fin de ilustrar los patrones de comercio de la industria - principalmente la integración comercial regional y la diversificación del mercado de exportación- en la Argentina y el Brasil durante el mismo período.

\section{Integración del Mercosur para la industria automotriz $^{10}$}

La integración de los países del Mercosur con relación a la industria automotriz aún no se ha alcanzado plenamente. Los países miembros no han logrado un acuerdo sobre el arancel externo común, las reglas del comercio intrabloque y la definición de las normas de origen. Hasta la fecha, no se ha acordado ningún régimen común. En su lugar existe una larga serie de acuerdos bilaterales. El Brasil y la Argentina firmaron el primer acuerdo en diciembre de 1994 y el más reciente en junio de 2008.

\footnotetext{
${ }^{9}$ Estadísticas de la Organización Internacional de Fabricantes de Automóviles.

${ }^{10}$ Esta sección se basa en gran parte en Arza y López (2008b).
}

El proceso de integración entre la Argentina y el Brasil pasó por cuatro etapas:

A la primera etapa se le llama de "no integración". Hasta 1994, en cada sistema regulatorio nacional se priorizaba la protección de los respectivos mercados internos. Las industrias de ambos países competían entre sí para ganar nuevos mercados internacionales.

La segunda etapa es llamada "hacia la integración". Este período va de 1995 al año 2000. A fines de 1994 ambos países firmaron el Protocolo de Ouro Preto, que definió las bases institucionales del Mercosur. Con algunas modificaciones, la Asociación Latinoamericana de Integración (ALADI) protocolizó este documento que se denominó Vigésimo octavo protocolo adicional del Acuerdo de Complementación Económica $\mathrm{N}^{\circ} 14$. Este acuerdo permitía a la Argentina y el Brasil continuar aplicando las normativas nacionales mientras no se llegara al desarrollo de una política común sobre el sector automotor para el Mercosur, prevista para el año 2000. Se preveía que en la política común se estableciera el libre comercio dentro del Mercosur, se llegara a un consenso sobre el arancel externo común y se abolieran los incentivos nacionales que distorsionaban la competitividad regional. Por lo tanto, mientras se trabajaba para alcanzar una política común, la Argentina y el Brasil acordaron:

i) El libre flujo entre ellos de automóviles y piezas, sujeto a los requisitos de desempeño establecidos en los respectivos sistemas regulatorios nacionales (las importaciones de un país socio debían compensarse con exportaciones a cualquier destino).

ii) Las partes y piezas importadas de los países del Mercosur, en la medida en que fueran compensadas por exportaciones, se consideraban nacionales para los efectos del cumplimiento de los estándares sobre contenido máximo importado.

iii) Se acordaron reglas específicas sobre cuotas comerciales — que se definieron por empresa- que no requerían compensación. El propósito de estas cuotas era, primero, compensar el déficit en que incurrió la Argentina entre 1991 y 1994 y, segundo, extender las preferencias arancelarias a las terminales automotrices ubicadas exclusivamente en alguno de los dos países. 
La tercera etapa se llamó de "profundización de la integración". Este período se extiende entre 2001 y 2005. El nuevo acuerdo se firmó en el año 2000 y se adoptó como Trigésimo primer protocolo adicional del Acuerdo de Complementación Económica $\mathrm{N}^{\circ}$ 14, que estuvo en vigor desde agosto de ese año hasta el 31 de diciembre de 2005.

En este acuerdo se establecía un arancel externo común del 35\% para las importaciones de vehículos automotores provenientes de terceros países, no sujetas a cuotas. Para los automóviles y utilitarios el arancel entró en vigor desde la firma del acuerdo, mientras que para otros productos automotores había un esquema que convergía en el 35\%, según se muestra en el cuadro 1.

Las partes y piezas se dividieron en tres grupos con diferentes niveles arancelarios, que convergían en 2005 en un $14 \%$, un $16 \%$ y un $18 \%$, respectivamente, como se detalla en el cuadro 1 . Cuando las partes y piezas no se producían en el país, las terminales automotrices podían importarlas con un arancel de solo un $2 \%$.

Con respecto al comercio intrabloque, a partir de enero 2001 los productos automotores estaban sujetos a una preferencia arancelaria del $100 \%$, siempre que se cumplieran las normas de origen (como se detalla más adelante) y que la proporción de importaciones y exportaciones de la industria entre socios no excediera los coeficientes comerciales aprobados para el bloque. Como se observa en el cuadro 1 , los coeficientes de desvío del comercio intrabloque (también llamados flex y definidos como la relación entre las importaciones y exportaciones entre los dos países) tendían a relajar las restricciones para el comercio en el interior del bloque con vistas a alcanzar el libre comercio hacia el año 2006.

Con el fin de beneficiarse del comercio preferencial intrabloque, las terminales automotrices deberían prever un contenido regional del $60 \%$. A los nuevos modelos se les permitía un contenido regional del $40 \%$ para el primer año y del 50\% para el segundo, pero debía alcanzarse el 60\% a partir del tercer año de producción. Sin embargo, una de las preocupaciones de la Argentina era que este acuerdo pudiera dañar su industria de partes y piezas, ya que el real brasileño estaba considerablemente subvaluado con respecto al peso argentino. Por lo tanto, el gobierno de la Argentina logró incluir una cláusula especial que se aplicaría a las subsidiarias localizadas en este país, en que se establecía un porcentaje máximo del $50 \%$ de partes y piezas importadas de cualquier destino hasta 2003, porcentaje que se elevaría al 60\% en 2004 $\mathrm{y}$ al $65 \%$ en 2005.

Por último, con el acuerdo se eliminaron los incentivos gubernamentales, puesto que toda la producción realizada a partir de incentivos promocionales o del

CUADRO 1

\section{Regulaciones para los productos automotores aplicables al comercio entre la Argentina y el Brasil, 2000-2006}

\begin{tabular}{|c|c|c|c|c|c|c|}
\hline \multirow{3}{*}{ Año } & \multicolumn{5}{|c|}{ Aranceles para el comercio extrabloque ${ }^{a}$} & \multirow{3}{*}{$\begin{array}{l}\text { Coeficiente de desvío (flex) } \\
\text { del comercio intrabloque } \\
\text { entre la Argentina y el Brasil } \\
\text { aprobado para una preferencia } \\
\text { arancelaria del } 100 \%\end{array}$} \\
\hline & \multirow{2}{*}{$\begin{array}{l}\text { Remolques y semirremolques, } \\
\text { camiones, camiones tractores y } \\
\text { chasis con motor de hasta } 5 \text { tone- } \\
\text { ladas de capacidad de carga }\end{array}$} & \multirow{2}{*}{$\begin{array}{l}\text { Ómnibus, carrocerías, camio- } \\
\text { nes, camiones tractores, chasis } \\
\text { con motor de hasta } 5 \text { toneladas } \\
\text { de capacidad de carga }\end{array}$} & \multicolumn{3}{|c|}{ Partes y piezas } & \\
\hline & & & I & II & III & \\
\hline 2000 & $25,0 \%$ & $18,0 \%$ & $7 \%$ & $8 \%$ & $9 \%$ & $\mathrm{~b}$ \\
\hline 2001 & $26,7 \%$ & $20,8 \%$ & $8,2 \%$ & $9,3 \%$ & $10,5 \%$ & 1,6 \\
\hline 2002 & $28,4 \%$ & $23,6 \%$ & $9,3 \%$ & $10,7 \%$ & $12,0 \%$ & 2 \\
\hline 2003 & $30,1 \%$ & $26,4 \%$ & $10,9 \%$ & $12,5 \%$ & $14,0 \%$ & 2,2 \\
\hline 2004 & $31,8 \%$ & $29,2 \%$ & $12,5 \%$ & $14,3 \%$ & $16,0 \%$ & 2,4 \\
\hline 2005 & $33,6 \%$ & $32,0 \%$ & $14,0 \%$ & $16,0 \%$ & $18,0 \%$ & 2,6 \\
\hline 2006 & $35,0 \%$ & $35,0 \%$ & c & c & c & Libre comercio \\
\hline
\end{tabular}

Fuente: elaboración propia sobre la base del Trigésimo primer protocolo adicional del Acuerdo de Complementación Económica No 14 , Asociación Latinoamericana de Integración (ALADI).

a Los términos dentro y fuera del bloque dentro del cuadro hacen referencia a la asociación entre la Argentina y el Brasil.

b El protocolo adicional mencionado en la Fuente regía a partir del 1 de agosto de 2000 en cuanto a tarifas, pero el flex regía a partir del 1 de enero de 2001. Esta normativa tenía vigencia hasta el 31 de diciembre del 2005. Por ello, no existe flex en el año 2000, pues el comercio intrabloque no estaba regulado para ese año.

c Si bien en la normativa se establece explícitamente que no existirá flex en 2006 (libre comercio intrabloque) y que el resto de los automotores alcanzarán niveles tarifarios iguales a los de los automóviles para ese año (del 35\%), la regulación con relación a las partes y piezas I, II, y III rige exclusivamente hasta el término de la vigencia de la norma (fines del 2005). Por eso, utilizando esa norma como fuente de información, no es posible completar las columnas I, II y III para el año 2006. 
apoyo de una instancia del gobierno se consideraba como producción extrabloque (aunque esta normativa no se aplicó retroactivamente).

A la cuarta etapa se la llamó de "retroceso en la integración". Al final del período cubierto por el acuerdo del año 2000 resultó claro que el nuevo gobierno argentino, en el poder desde 2003, no consideraba deseable avanzar hacia un régimen de libre comercio regional. Por consiguiente, tras largas negociaciones se firmó un nuevo acuerdo en junio de 2006 (el Trigésimo quinto protocolo adicional del Acuerdo de Complementación Económica $\mathrm{N}^{\circ} 14$ ), cuyo efecto se extendió hasta junio de 2008. En este acuerdo se estableció una serie de regulaciones que cumplían en gran parte con el acuerdo anterior; sin embargo, se introdujeron cambios en el comercio intrabloque. En lugar de alcanzar el libre comercio, con el acuerdo firmado en junio de 2006 se estableció un coeficiente de desvío más restrictivo equivalente a 1,95 —el vigente en 2005 era 2,6 (véase el cuadro 1). En 2008 se firmó un nuevo acuerdo (Trigésimo octavo protocolo adicional del Acuerdo de Complementación Económica No 14), válido hasta 2014. En este se confirmó que el coeficiente de desvío 1,95 sería válido cuando la Argentina tuviera un déficit en su comercio automotriz con el Brasil, pero que aumentaría a 2,5 cuando ocurriera lo contrario (por ejemplo, el comercio intrabloque es más restringido cuando los déficits afectan a la Argentina). No se prevé que se alcance el libre comercio intrabloque sino hasta julio de 2013.

En este estudio se completa el análisis empírico de 2005, es decir, antes del inicio de la etapa de "retroceso en la integración".

Por último, la influencia regional de la industria automotriz del Mercosur se ha estado expandiendo gracias a diversos acuerdos preferenciales de comercio firmados con otros países de América Latina desde fines de la década de 1990: Chile (1996 y 2002), México (2003) y la República Bolivariana de Venezuela, Colombia y el Ecuador (2005). La Argentina y el Brasil también firmaron diversos acuerdos con el Uruguay desde la década de 1980; sin embargo, en el contexto de la integración del Mercosur se dieron pasos importantes para el sector automotor, primero en 1994, más tarde en el año 2002 (el Brasil) y en 2003 (la Argentina).

\section{Patrones de comercio de la industria automotriz de la Argentina y el Brasil}

Con frecuencia se considera al sector automotor como un importante pilar del desarrollo económico e industrial en la Argentina y el Brasil, y ha tenido el apoyo sistemático de gobiernos con puntos de vista opuestos sobre la política económica. Hasta cierto punto, este apoyo correspondió a cuestiones de economía política (existen intereses creados a lo largo de la extensa historia de producción del sector que hacen difícil quitarle o reducirle el apoyo gubernamental). No obstante, su importancia económica es innegable. En 2005, la industria automotriz y de piezas representaba el 5,3\% del valor bruto de la producción industrial de la Argentina y el $3,5 \%$ del empleo industrial, mientras que en el Brasil estas cifras eran aún más destacadas: un 10,9\% y un $6,2 \%$, respectivamente.

El crecimiento de la producción automotriz en la Argentina ha sido errático (véase el gráfico 2). La industria produjo menos automóviles en 1990 que en 1961. En la década de 1990 se registraron puntos elevados de crecimiento, pero en el año 2002 la producción retrocedió a niveles similares a los de 1964. Tal comportamiento errático en la Argentina, comparado con el del Brasil, explica por qué la producción brasileña de automóviles, que fue similar a la de la Argentina hasta mediados de los años sesenta, era seis veces superior en 2006.

El desempeño de las exportaciones también es muy asimétrico en ambos países (véase el gráfico 3). No fue sino hasta 2005 que las exportaciones de automotores de la Argentina alcanzaron a las que el Brasil tenía a comienzos de la década de 1990 (alrededor de 180.000 unidades). Desde entonces las exportaciones brasileñas siguieron creciendo y en 2006 el Brasil exportaba 3,6 veces más que la Argentina (véase el gráfico 3). Las exportaciones argentinas aumentaron notablemente en la década de 1990 (entre 1992 y 2001 lo hicieron a una tasa anual acumulada del $28 \%$ ), pero una vez más la recesión y la crisis se tradujeron en un retroceso del desempeño exportador en 2002 y 2003. Recién en 2004 las exportaciones comenzaron a crecer nuevamente de manera significativa.

El desempeño registrado por el Brasil respecto del acceso a mercados extrabloque también superó al de la Argentina. Como se constata al comparar los gráficos 4 y 5 , en el Brasil se logró diversificar el mercado de exportación en mayor medida que en la Argentina. Este último país comenzó a exportar por fuera del Mercosur en 2002, pero lo hizo sobre todo hacia América Latina. En cambio, el Brasil llegó a mercados más exigentes, como los de Europa y América del Norte a comienzos de la década de 1990. Aunque estos mercados aún representan una participación minoritaria en las exportaciones totales del Brasil (alrededor del 16\% en 2005), no debe restarse importancia a su gravitación económica: en 2005 el Brasil exportó a Europa y América del Norte 
GRÁFICO 2

Argentina y Brasil: incremento de la producción automotriz, 1959-2006 (En miles de unidades)

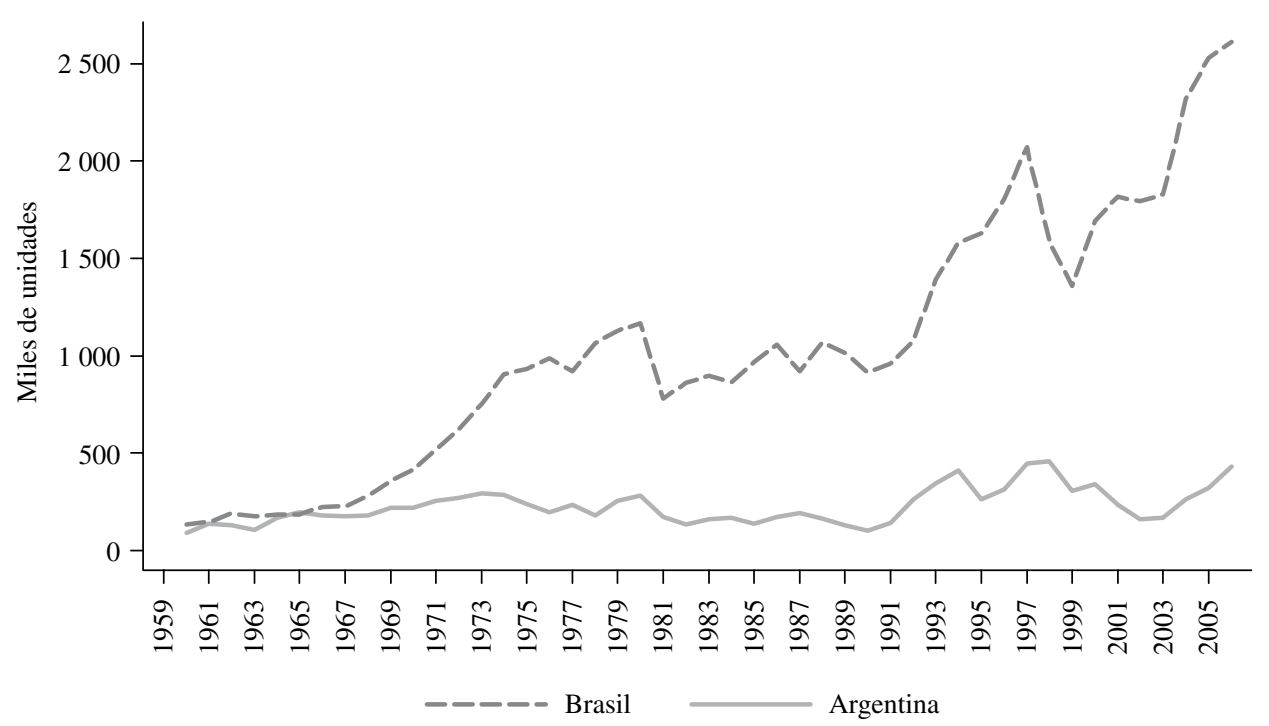

Fuente: elaboración propia sobre la base de la Asociación de Fábricas de Automotores (ADEFA) y la Asociación Nacional de Fabricantes de Vehículos Automotores (ANFAVEA).

GRÁFICO 3 Argentina y Brasil: total de exportaciones del sector automotor, 1990-2006 (En unidades)

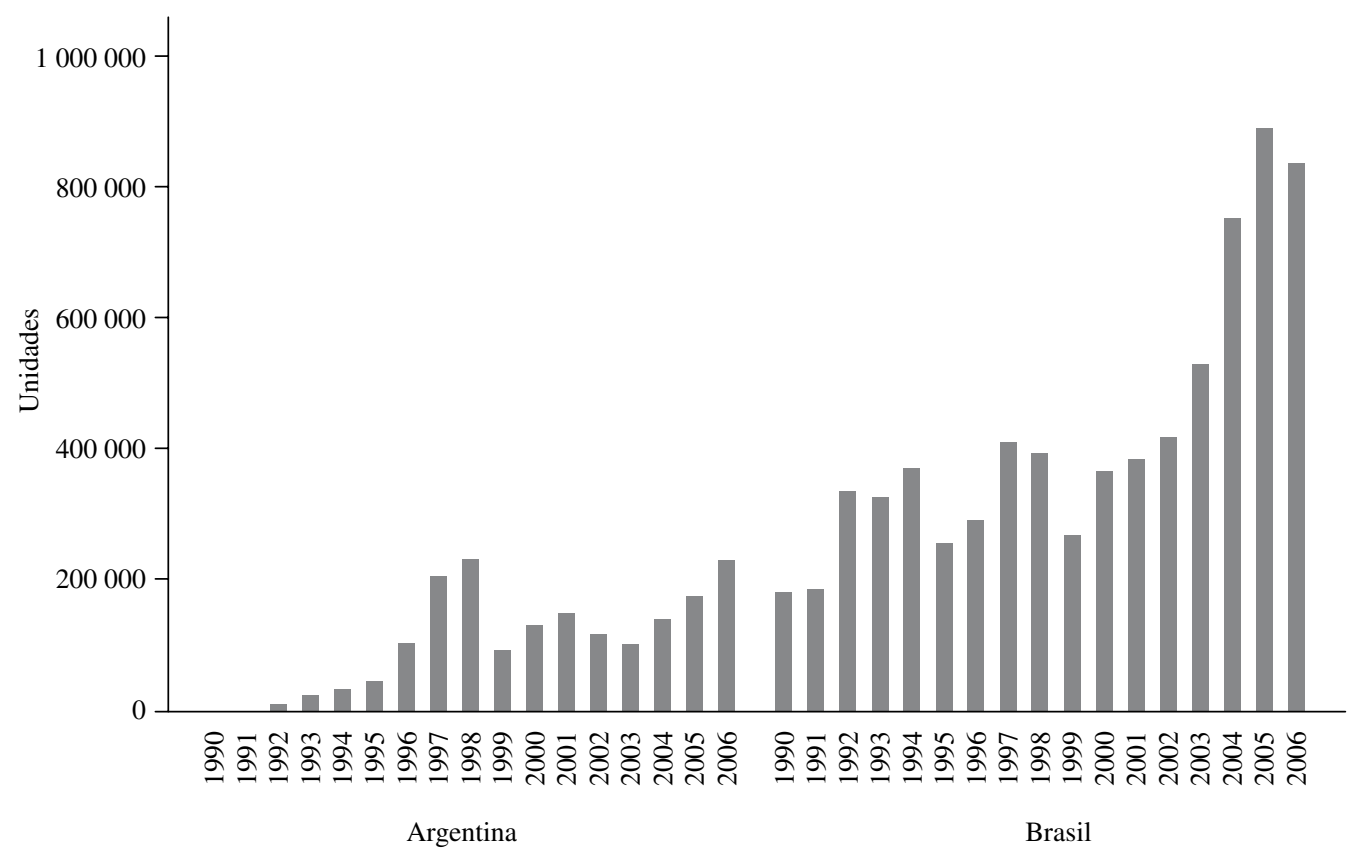

Fuente: elaboración propia sobre la base de Asociación de Fábricas de Automotores (ADEFA) y Asociación Nacional de Fabricantes de Vehículos Automotores (ANFAVEA). 
GRÁFICO 4

Brasil: total de exportaciones del sector automotor, por destino, 1991-2005

(En millones de dólares del año 2000)

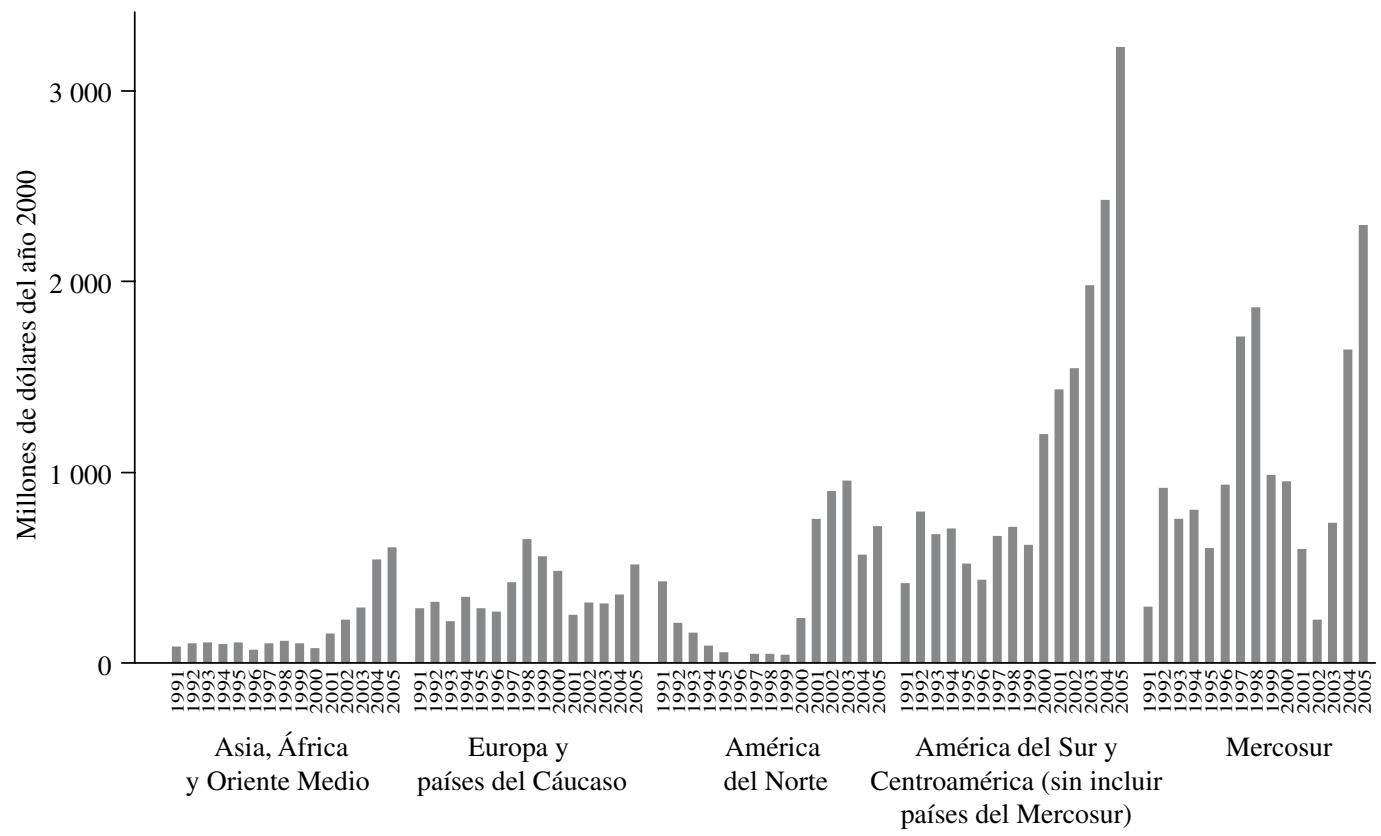

Fuente: elaboración propia a partir de la Base de datos estadísticos sobre el comercio de mercaderías (COMTRADE) de las Naciones Unidas.

GRÁFICO 5

Argentina: total de exportaciones del sector automotor, por destino, 1991-2005

(En millones de dólares del año 2000)

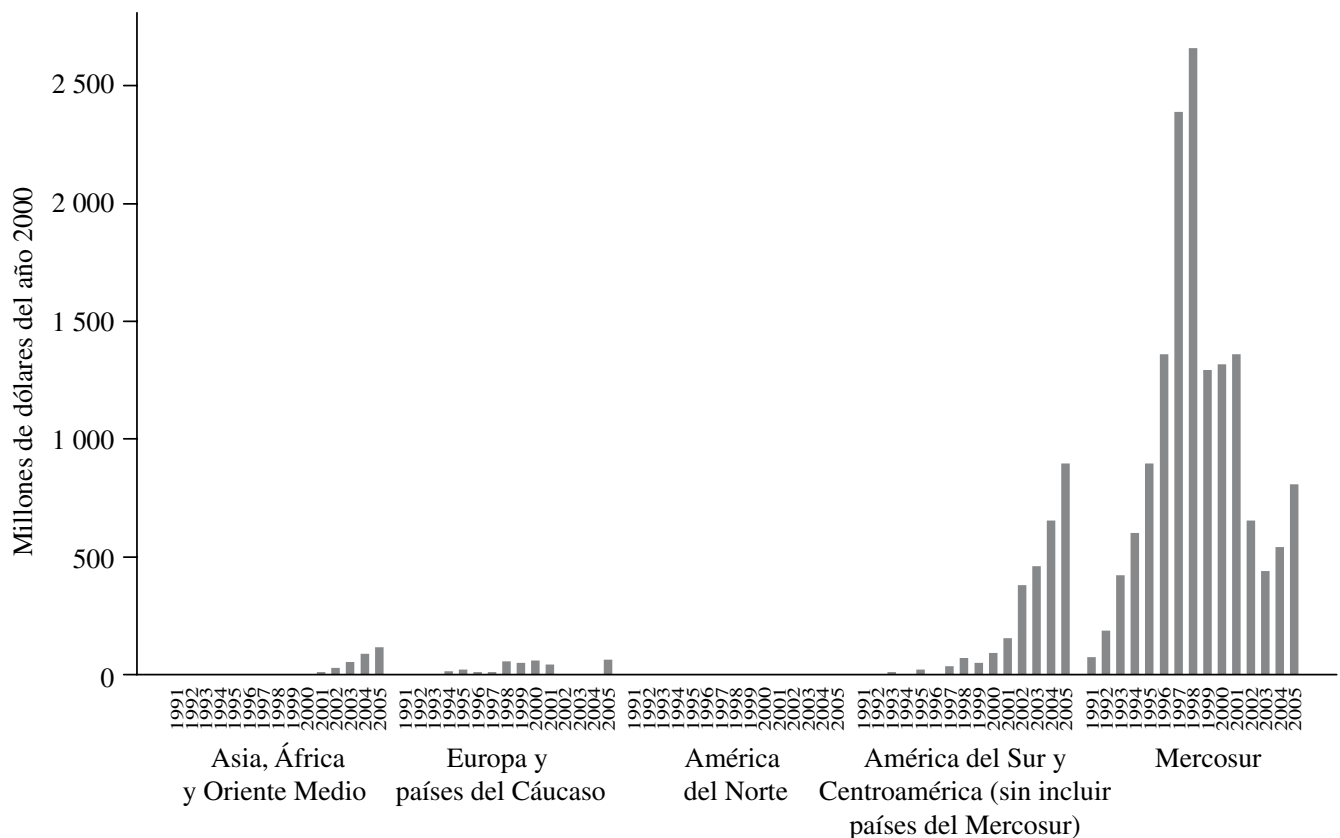

Fuente: elaboración propia a partir de la Base de datos estadísticos sobre el comercio de mercaderías (COMTRADE) de las Naciones Unidas. 
una cantidad de automotores que fue tan solo un 35\% menor que la del total de exportaciones de la Argentina (a todo el mundo) en el mismo año.

En el gráfico 6 se muestran las exportaciones de automotores (CIIU Rev. 2 N$^{\circ} 341$ ) de la Argentina y el Brasil (Naciones Unidas, 1986, pág. 341) a mercados con y sin acuerdos preferenciales de comercio (es decir, exportaciones al Mercosur, Chile, México, Colombia, el Ecuador y la República Bolivariana de Venezuela comparadas con exportaciones a otros mercados). De este gráfico surge lo siguiente:

i) el comercio intrabloque del Mercosur se incrementó sustancialmente en 1995, despegando más rápido en el caso de la Argentina,

ii) el desorden macroeconómico de 1998-1999 (recesiones en la Argentina y el Brasil) y de 2001-2002 (crisis de la Argentina) repercutió negativamente en el comercio intrabloque; ${ }^{11}$

\footnotetext{
${ }^{11}$ De hecho, el comercio intrabloque es muy elástico respecto del producto interno bruto (PIB) de la Argentina (la elasticidad de las exportaciones con respecto al PIB argentino, es de alrededor de 7 para las exportaciones argentinas y alrededor de 6 para las brasileñas), pero es bastante inelástico en relación con el PIB del Brasil.
}

iii) México y Chile se volvieron importantes mercados para la Argentina, pero sobre todo para el Brasil alrededor del período 2000-2003;

iv) Colombia, el Ecuador y la República Bolivariana de Venezuela no son mercados importantes para la Argentina;

v) otros mercados sin acuerdos preferenciales de comercio siempre han sido muy relevantes para las exportaciones del Brasil y comenzaron tímidamente a recibir exportaciones de la Argentina en 2004-2005.

En el gráfico 7 se muestra la cantidad total de mercados a los que la Argentina y el Brasil exportaron desde 1991. Como puede verse, la Argentina exportó automóviles solo a 12 mercados en 1991, pero hacia 2005 esa cifra se quintuplicó (64 mercados). El Brasil ya había exportado a muchos más mercados en 1991 (93) y en 2005 la cifra llegaba a 130 destinos. Durante la etapa de "profundización de la integración" ambos países quebraron su tendencia y llegaron a una cifra de mercados mucho más importante que antes (la Argentina en 2001 y el Brasil en 2002).

Sin embargo, la diversificación de los mercados supone no solo acceder a nuevos mercados, sino también

GRÁFICO 6

Argentina y Brasil: exportaciones de automóviles a mercados con y sin acuerdos preferenciales de comercio, 1991-2005

(En millones de dólares del año 2000)

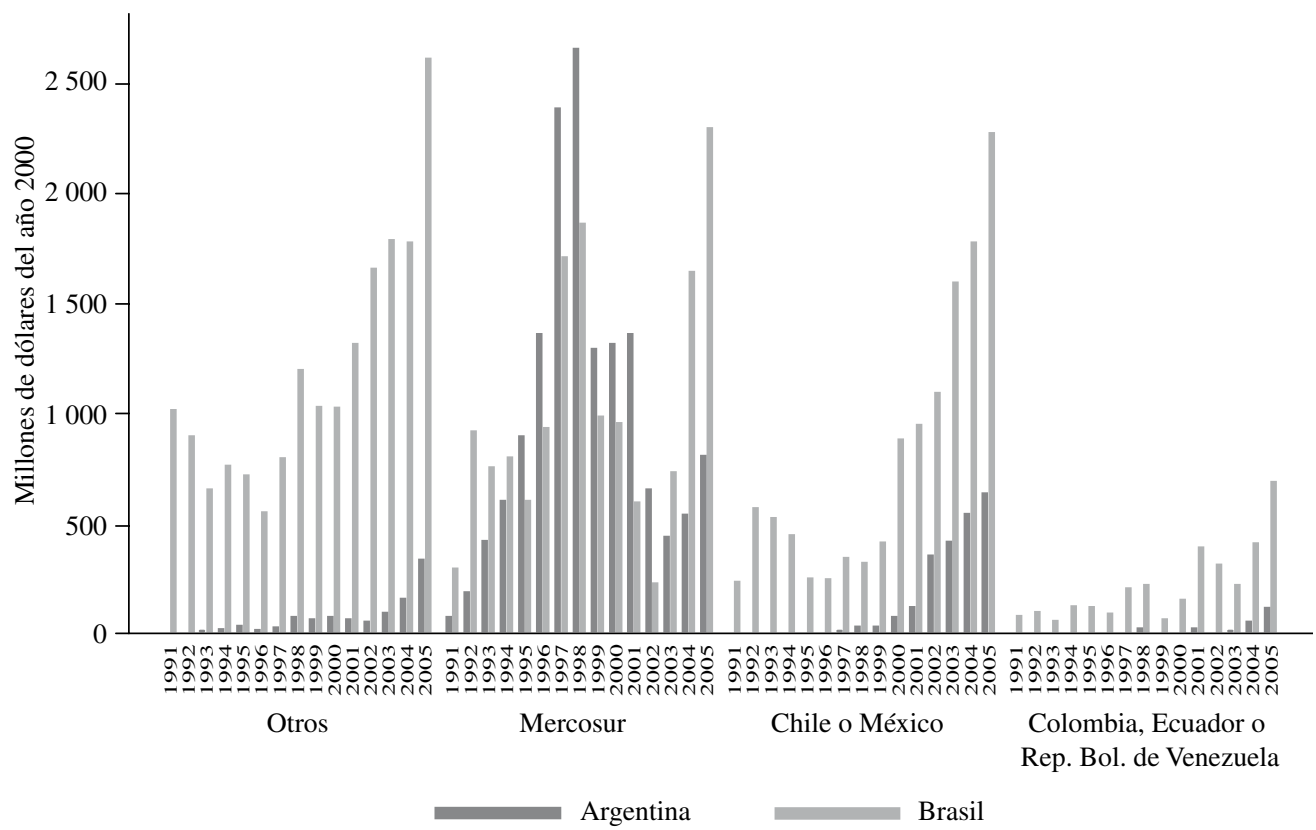

Fuente: elaboración propia a partir de la Base de datos estadísticos sobre el comercio de mercaderías (COMTRADE) de las Naciones Unidas. 


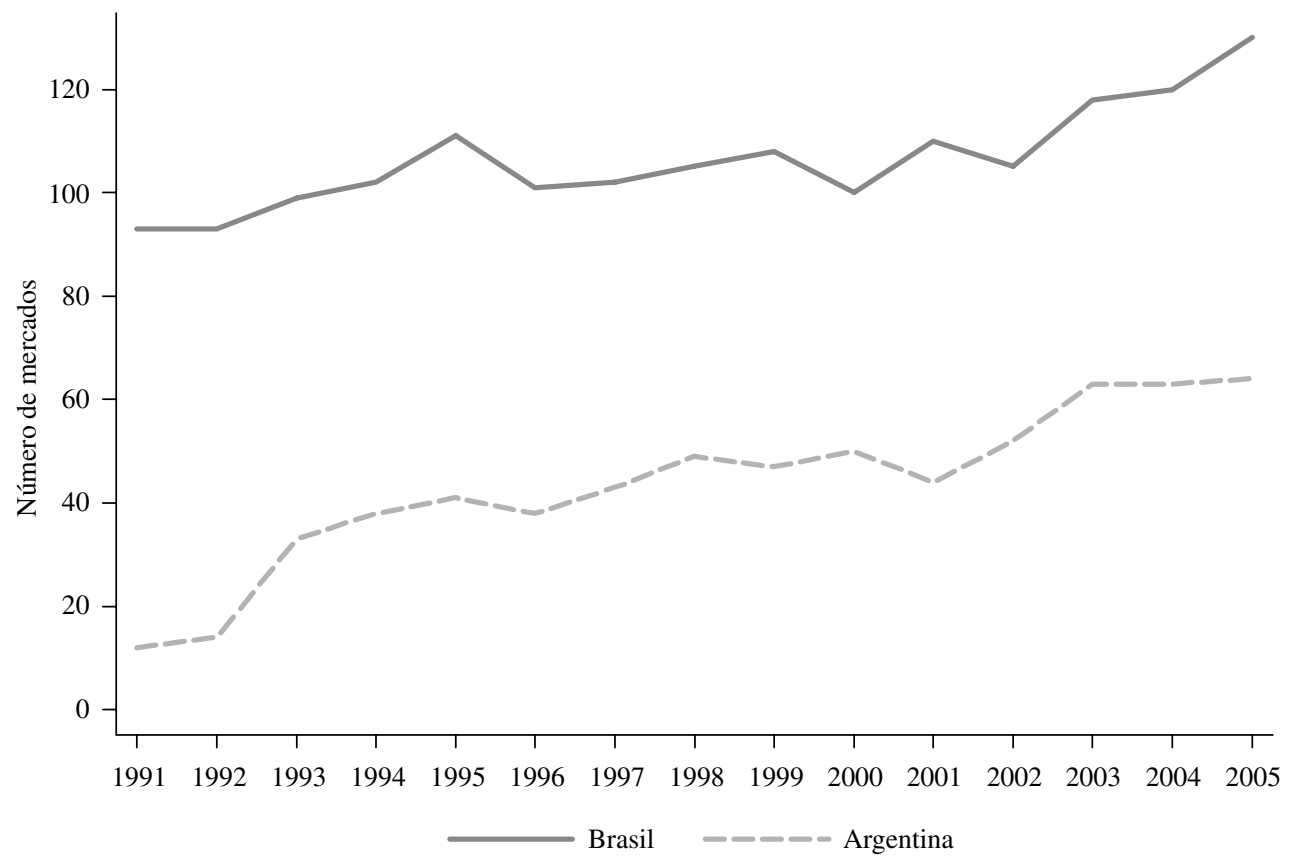

Fuente: elaboración propia a partir de la Base de datos estadísticos sobre el comercio de mercaderías (COMTRADE) de las Naciones Unidas.

exportar en niveles similares a todos ellos. De hecho, en 2005 el $40 \%$ de las exportaciones de automóviles de la Argentina iban a un solo país, el Brasil, mientras que ese año el $28 \%$ del total de las exportaciones del Brasil estaban destinadas a la Argentina. En otras palabras, la cantidad vendida a cada mercado estaba lejos de ser equilibrada, especialmente en el caso de la Argentina. Para representar este aspecto de la diversificación se elabora un índice equivalente para cada país, que se define como:

$$
F=\frac{1}{\sum_{j}^{n} F_{j}^{2}}
$$

donde $F_{j}$ es la fracción de las exportaciones totales de la Argentina o del Brasil que va a cada mercado $j$. El índice tiene un valor mínimo de 1 cuando las exportaciones totales de la Argentina o del Brasil se venden a un mercado único. De lo contrario, el índice equivalente evalúa la diversificación en términos de número de mercados con iguales participaciones en las exportaciones. Por ejemplo, en el gráfico 8 se ve que la diversificación de las exportaciones de la Argentina en 2005 es equivalente a la diversificación de un país que exporta participaciones iguales a cuatro mercados. ${ }^{12}$

En el gráfico 8 se observa además que la diversificación era más o menos estable en la Argentina hasta 2001. En el período 1991-1994 (etapa de "no integración") el índice era de alrededor de 1,5. En el período 1995-2000 (etapa "hacia la integración") era un poco más bajo $(1,2)$ y aumentó a alrededor de 3,1 en el período 2001-2005 (fase de "profundización de la integración"). En otras palabras, las exportaciones de la Argentina se concentraron en gran medida en el Brasil antes y después del primer acuerdo; sin embargo, durante la fase de "profundización de la integración" la industria llegó a nuevos mercados, sobre todo Chile y México (véase el gráfico 6).

\footnotetext{
${ }^{12}$ El número absoluto del índice equivalente $F$ se determina por el número total de mercados a los que exportó cada país. Por lo tanto, dado que el Brasil exporta a más mercados se espera que el índice equivalente sea mayor. Podría haberse estandarizado el índice por el número total de mercados, pero la idea fue representar no solo participaciones iguales en los mercados, sino también el número de mercados que cada país alcanzó.
} 


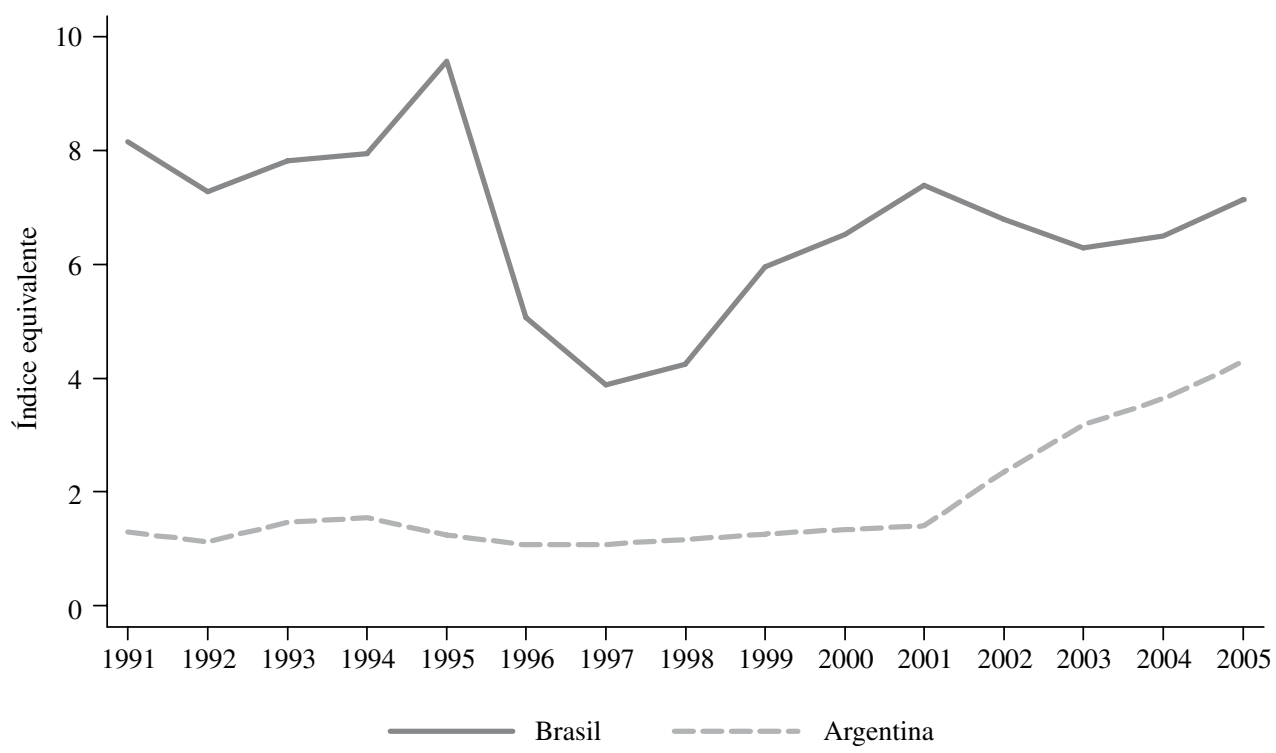

Fuente: elaboración propia a partir de la Base de datos estadísticos sobre el comercio de mercaderías (COMTRADE) de las Naciones Unidas.

a Significa que el índice puede adoptar valores entre 1 e infinito.

En cambio, en el Brasil la diversificación disminuyó drásticamente después del primer acuerdo (durante la fase "hacia la integración"). El mercado argentino comenzó entonces a ser el prioritario. Por ejemplo, en 1991 el 12\% de las exportaciones del Brasil se dirigían a la Argentina y otro 12\% se destinaba a Chile. En 1996 las exportaciones a la Argentina representaron casi el $41 \%$ del total, mientras que las exportaciones a Chile continuaron ubicándose en alrededor del $12 \%$. Sin embargo, desde 1997 la participación de la Argentina disminuyó y simultáneamente el Brasil penetró en nuevos mercados. Por lo tanto, el índice de diversificación aumentó. En 2005, el Brasil alcanzó un índice equivalente de alrededor de 7, que todavía era inferior al de 1991 (alrededor de 8), aunque el país había logrado un 25\% más de mercados en 2005 que en 1991.

En suma, el Brasil superó a la Argentina en producción, exportaciones y diversificación del mercado de exportación. El índice equivalente sugiere que proporciones similares de sus exportaciones se destinan a más mercados que lo que logra la Argentina, país que en cambio sigue dependiendo en gran medida de mercados con acuerdos preferenciales de comercio.

Existen diversos factores que explican la diferencia entre los patrones de desarrollo de la industria automotriz en la Argentina y el Brasil. En primer lugar, desde el punto de vista macroeconómico, el clima de negocios era más predecible en el Brasil que en la Argentina en el período 1989-2005 y las exportaciones (e importaciones) de la Argentina se vieron mucho más afectadas por los ciclos macroeconómicos que las del Brasil. En segundo lugar, el sistema regulatorio se diseñó y aplicó de manera diferente y supuso un grado asimétrico de ayuda económica en cada país. Aunque el sector automotor fue apoyado por ambos gobiernos, en el Brasil la política de apoyo ha sido mucho más directa y sistemática desde los orígenes de la industria. Instituciones federales, provinciales e incluso municipales han ofrecido subsidios y créditos blandos (Laplane y Sarti, 2008; Motta Veiga, 2004; Oman, 2000). En cambio, en la Argentina las regulaciones fueron bastante discrecionales y a veces inconsistentes, lo que se sumó a la falta de previsibilidad de las tendencias macroeconómicas. Además, el gobierno rara vez exigió el cumplimiento de los compromisos que las empresas habían asumido en diferentes momentos en cuanto, por ejemplo, al desempeño exportador. Asimismo, la atención de la política al desarrollo de la cadena de valor fue muy difusa, como débil fue la motivación para que las subsidiarias llevaran a cabo actividades innovadoras en el país (véanse más detalles en Arza y López, 2008c). 
En tercer lugar, había diferencias estructurales entre las industrias de la Argentina y el Brasil. El mercado brasileño era por lo menos cuatro veces más grande y su red industrial estaba más desarrollada. Esto podría sugerir que estos dos países le otorgaron diferente importancia estratégica a la industria. Además, el tamaño del mercado interno le permitió históricamente a las subsidiarias localizadas en el Brasil alcanzar mejores escalas de producción - promoviendo entonces su eficiencia productiva - que las logradas por las subsidiarias localizadas en la Argentina. Humphrey y Oeter (2000, pág. 63) sostienen que puede considerarse eficiente una escala mayor de 50.000 unidades para el ensamble de vehículos livianos. En 1999, en la Argentina se producían 27 modelos diferentes de vehículos livianos y ninguno de ellos en un volumen mayor de 35.000 unidades. En cambio, en el Brasil se producían 44 modelos y seis de ellos a escala eficiente. En 2006, en la Argentina se producían 17 modelos y dos de ellos a escala eficiente, mientras que en el Brasil se producían 43 modelos, 15 de ellos a escala eficiente. El mejor uso de las escalas en el Brasil se relaciona con el tamaño de su mercado, dado que - en promedio - el 68\% de la producción de cada modelo se vendía en el mercado interno en 2006; en la Argentina, en cambio, el mercado interno absorbía en promedio un $44 \%$ de la producción de cada modelo.

En suma, la evidencia permite constatar, en general, que el desempeño de la industria automotriz de la Argentina fue diferente de la del Brasil. Sin embargo, el propósito de este artículo es comprender en qué medida los esfuerzos a nivel regional de las empresas y los gobiernos podrían producir incrementos de competitividad en ambos países. En las secciones IV y V que siguen se presenta el plan de investigación empleado con ese fin.

\section{IV}

\section{Preguntas de investigación e hipótesis}

Los datos empíricos antes señalados parecen indicar que el comercio intrabloque se incrementó durante el período en que se inició la integración en 1994. En el caso del Brasil, esto podría haber ocurrido a costa del comercio extrabloque. En el caso de la Argentina, la evidencia descriptiva sugiere que se creó genuinamente comercio después del primer acuerdo.

Además, en ambos países parece haberse diversificado el mercado de exportación durante la etapa de "profundización de la integración" (2000-2005). Esto respaldaría la hipótesis de que el Mercosur se transformó en una plataforma de producción y exportación como consecuencia de sus políticas regionales. Sin embargo, si bien en la Argentina el fenómeno de la diversificación comenzó recién durante ese período, el Brasil mostró un patrón histórico de diversificación creciente brevemente interrumpido tras el primer acuerdo (1994). Asimismo, en la Argentina la diversificación se debió en mayor medida a las exportaciones a los mercados con los que se tenían acuerdos preferenciales de comercio, no siendo este el caso del Brasil. Una explicación alternativa para la diversificación del mercado de exportación es que la recesión macroeconómica en ambos países los impulsó a buscar nuevos mercados para colocar su exceso de producción.

En este trabajo se examina el papel de los acuerdos del Mercosur (en este caso, entre la Argentina y el
Brasil) para la creación de comercio y la diversificación del mercado de exportación.

Las preguntas de investigación son:

¿Hay evidencia de creación de comercio después de 1994? ¿Y después del año 2000? ¿Existen patrones de diversificación del mercado de exportación después de esas fechas? ¿Estos patrones son similares en los casos de la Argentina y el Brasil? ¿La diversificación surgió a expensas del comercio intrabloque?

$\mathrm{Al}$ respecto, se plantean las siguientes hipótesis:

- Hipótesis 1: Tras el primer acuerdo del Mercosur para la industria automotriz (1994) hubo creación de comercio en la Argentina y el Brasil.

- Hipótesis 2: Tras el segundo acuerdo del Mercosur para la industria automotriz (año 2000) hubo creación de comercio en la Argentina y el Brasil.

- Hipótesis 3.1: El Brasil, la Argentina o ambos han diversificado sus exportaciones a mercados extrabloque desde la firma de su segundo acuerdo comercial en el año 2000.

- Hipótesis 3.2: La diversificación desde el acuerdo comercial del año 2000 ocurrió a expensas de las exportaciones intrabloque (es decir, se relacionó con la contracción de la demanda de los socios intrabloque debida a la recesión macroeconómica en sus países). 


\section{V}

\section{Metodología}

\section{El modelo de gravedad}

En este artículo se sigue la metodología empleada en la literatura de la integración con que se intenta medir el grado de creación y desvío de comercio a partir de diferentes acuerdos institucionales (Aitken, 1973; Bayoumi y Eichengreen, 1997; Braga, Safadi y Yeats, 1994; Frankel, 1997; Krueger, 1999; Soloaga y Winters, 2001).

Los modelos de gravedad se inspiran en las leyes de la física relativas a la atracción de los objetos según su masa y la distancia entre ellos.

$$
\text { fuerza_de_gravedad }=G \frac{M_{i} M_{j}}{\left(\text { dist }_{i j}\right)^{2}}
$$

En teoría de comercio, la atracción física se sustituye por la atracción comercial que, según se dice, es dependiente del tamaño del país y la distancia entre países $(G$ es un término constante). El tamaño se define de acuerdo con el tamaño del mercado del importador y la capacidad de producción del exportador. La distancia, a su vez, se define por las barreras (institucionales y geográficas) y la distancia (geográfica y cultural). Por lo tanto, los modelos de gravedad básicos se definen como:

$$
\begin{aligned}
& X_{i j}=\alpha+\beta_{1} Y_{i}+\beta_{2} N_{i}+\beta_{3} P C_{j}+\beta_{4} N_{j}+ \\
& \beta_{5} T_{i}+\beta_{6} T_{j}+\beta_{7} A D_{i}+\beta_{8} D_{i j}+\beta_{9} A_{i j}+ \\
& \beta_{10} I_{i}+\beta_{11} I_{j}+\beta_{12} L L_{i}+\beta_{13} L L_{j}+ \\
& \beta_{14-18} C L_{i j}+\varepsilon_{i j}
\end{aligned}
$$

donde:

$i \quad$ es el país importador y $j$ el país exportador.

$X_{i j}=$ importaciones (en miles de dólares a precios constantes del año 2000) del país $i$ provenientes del país $j$ (en logaritmos naturales).

$Y_{i}=\quad$ PIB del país importador (en dólares a precios constantes del año 2000) (en logaritmos naturales).

$N=$ población del país importador/exportador (en logaritmos naturales).

$T=$ área de territorio del país importador/exportador (en logaritmos naturales).

$P C_{j}=$ capacidad de producción del país exportador, definida como la producción máxima de los cinco años anteriores en dólares a precios del año 2000 (en logaritmos naturales).

$A D_{i}=$ distancia media entre el país $i$ y todos sus socios de exportación, ponderada por los flujos de comercio (medida de alejamiento) (en logaritmos naturales).

$D_{i j}=\quad$ distancia entre el país $i$ y el país $j$ en kilómetros (en logaritmos naturales).

$A_{i j}=$ variable dicotómica para países vecinos $i j$.

$I=\quad$ variable dicotómica para países insulares.

$L L=$ variable dicotómica para países sin litoral.

$C L_{i j}=$ variable dicotómica para idioma común entre países $i j$. Esta se subdivide en cinco variables dicotómicas para diferentes idiomas (árabe, español, francés, inglés y otros).

Estos modelos se han ampliado para abarcar otros aspectos que afectan al comercio entre países y que no se relacionan con el tamaño y la distancia, por ejemplo, mediante variables dicotómicas para bloques comerciales, indicadores de ventajas comparativas reveladas o la evolución de los tipos de cambio bilaterales, entre otros (Filippini y Molini, 2003, Musila, 2005, Soloaga y Winters, 2001).

El modelo ampliado que se usa en este estudio es:

$$
\begin{aligned}
& X_{i j}=\alpha+\beta_{1} Y_{i}+\beta_{2} N_{i}+\beta_{3} P C_{j}+\beta_{4} N_{j}+\beta_{5} T_{i}+ \\
& \beta_{6} T_{j}+\beta_{7} A D_{i}+\beta_{8} D_{i j}+\beta_{9} A_{i j}+\beta_{10} I_{i}+\beta_{11} I_{j}+ \\
& \beta_{12} L L_{i}+\beta_{13} L L_{j}+\beta_{14-18} C L_{i j}+\beta_{19} R C A_{j j}+ \\
& \beta_{20-34} B L O C_{i j}+\varepsilon_{i j}
\end{aligned}
$$

donde se agregaron las siguientes variables a la ecuación básica (2):

$R C A_{i j}=$ ventaja comparativa manifiesta, definida como la relación entre la $R C A$ del país $i$ en relación con la $R C A$ del país $j . R C A_{i}$ se define como la participación del país $i$ en las exportaciones mundiales de automóviles con respecto a la participación del país $i$ en relación con las exportaciones mundiales de todos los productos comercializados. Cuando el indicador es mayor que 1 , se dice que el país $i$ tiene una ventaja comparativa en la producción de automóviles. 
Con esta variable se intenta medir la razón de competitividad en la producción de automóviles entre el importador y el exportador, y se espera que afecte negativamente a la cantidad de vehículos importados.

$B L O C_{i j}=$ variables dicotómicas que representan los flujos bilaterales en 15 bloques comerciales (véase el anexo 1). Estas variables dicotómicas pueden considerarse ayudas institucionales para acortar las distancias entre los países; en otras palabras, se espera que los países que integran los bloques comercien más entre sí.

Con el fin de cuantificar si la creación o el desvío de comercio existieron en diferentes circunstancias, se emplea un conjunto de variables dicotómicas con que se identifica el comercio desde, hacia o entre grupos de socios. Esta metodología fue propuesta originalmente por Aitken (1973). Desde entonces abundaron los estudios empíricos en que se empleaba y mejoraba la metodología original.

En este artículo se emplea la metodología propuesta por Soloaga y Winters (2001). Recordemos que el objetivo es probar si se creó comercio después de un evento en particular (como la firma de los acuerdos de 1994 y 2000). Este método se propone incluir tres variables dicotómicas: una primera que identifica el bloque cuando sus miembros importan de fuentes extrabloque; una segunda que identifica el bloque cuando exporta hacia destinos extrabloque, y una tercera variable que identifica el comercio intrabloque. Para evaluar si hubo creación de comercio deben compararse los coeficientes de estas variables después y antes del evento: habrá creación de comercio cuando el incremento en la tercera variable sea mayor que la disminución de la primera variable; por el contrario, habrá desvío de comercio cuando estos dos efectos sean similares.

\section{Fuentes de datos y cobertura}

Se usa la Base de datos estadísticos sobre el comercio de mercaderías (COMtrade) (CIIU Rev. 2 No 341) de las Naciones Unidas, que cubre flujos comerciales bilaterales de la industria automotriz de 1989 a 2006 (Naciones Unidas, 1986, pág. 341). Para construir la base de datos empleada en este trabajo se tomaron como primera opción los flujos de importación y, en los casos en que faltara información, esta se completó utilizando datos de los flujos de exportaciones. Sin embargo, la base de datos COMTRADE tiene una cobertura diferente para distintos años, faltando datos durante los primeros años y también en el último período comprendido. Por lo tanto, se acortó el período para abarcar solo los años en que los datos de la Argentina y el Brasil estuvieran razonablemente completos (1991-2005). ${ }^{13}$

Para cumplir con los requerimientos de información que demandan los modelos de gravedad se emplearon:

i) La base de datos del Banco Mundial sobre comercio, producción y protección, que contiene información para todas las variables independientes de la ecuación (2) respecto de 100 países durante el período 1970-2004, con excepción de la capacidad de producción.

ii) Los Indicadores del Desarrollo Mundial del Banco Mundial, para actualizar la información variable en el tiempo hasta el año 2005.

iii) La base de datos de estadísticas industriales de la Organización de las Naciones Unidas para el Desarrollo Industrial (ONUDI), a fin de elaborar el indicador de capacidad de producción.

iv) Conjuntos de datos estadísticos de la Organización Mundial del Comercio (OMC) para identificar los acuerdos de integración regional.

v) Datos sobre información jurídica de los ministerios de economía de la Argentina y el Brasil para identificar los acuerdos preferenciales de comercio con terceros países y otra información sobre regulación que afecta a la industria automotriz en ambos países.

Dado que la disponibilidad de información era diferente en cada base de datos utilizada, para estimar las ecuaciones finalmente se logró construir un panel de datos desbalanceado de 59.165 flujos bilaterales entre $1991 \mathrm{y}$ 2005 (entre 3.393 y 4.163 flujos bilaterales por año).

\section{Prueba de las hipótesis}

Para someter a prueba las hipótesis mencionadas en la sección IV, la muestra se dividió en tres períodos. El primer período va desde 1991 hasta 1994 y representa la etapa de "no integración"; el segundo período transcurre desde 1995 hasta el año 2000 y abarca íntegramente la etapa "hacia la integración". Por último, el tercer período comprende desde el año 2001 hasta el 2005 y representa toda la etapa de "profundización de la integración".

Se estimaron dos modelos diferentes para someter a prueba las hipótesis antes mencionadas.

\footnotetext{
${ }^{13}$ Se usaron los datos comerciales producidos por las oficinas nacionales de estadísticas de la Argentina y el Brasil para comprobar la completitud de la base de datos COMTRADE.
} 


\section{Modelo 1}

Se utiliza para testear las hipótesis 1 y 2 . Se elaboraron tres variables dicotómicas, tal como proponen Soloaga y Winters (2001), y se agregaron a la ecuación (3).

ARGBRA $_{i j}=$ es la variable dicotómica que identifica los flujos de comercio entre la Argentina y el Brasil.

ARGBRA $_{i}=$ es la variable dicotómica que identifica otras importaciones de la Argentina y el Brasil.

ARGBRA $_{j}=$ es la variable dicotómica que identifica las exportaciones de la Argentina y el Brasil a otros destinos.

La hipótesis 1 será verdadera si hay un incremento significativo del coeficiente de ARGBRA $_{i j}$ entre los períodos $1^{\circ}$ y $2^{\circ}$ que no se compense con una disminución del coeficiente de ARGBRA $_{i}$

La hipótesis 2 será verdadera si hay un aumento significativo del coeficiente de ARGBRA $_{i j}$ entre los períodos $2^{\circ}$ y $3^{\circ}$ que no se compense con una reducción del coeficiente de ARGBRA $_{i}$.

\section{Modelo 2}

Se emplea para testear las hipótesis 3.1 y 3.2. Las variables dicotómicas incluidas son las siguientes:

ARGBRA $_{i j}=$ es la variable dicotómica que identifica los flujos de comercio entre la Argentina y el Brasil.

$\mathrm{ARGCHL}_{i j}=$ es la variable dicotómica que identifica los flujos de comercio entre la Argentina y Chile.

$\mathrm{ARGMEX}_{i j}=$ es la variable dicotómica que identifica los flujos entre la Argentina y México.

ARGURY $_{i j}=$ es la variable dicotómica que identifica los flujos de comercio entre la Argentina y el Uruguay.

BRACHL $_{i j}=$ es la variable dicotómica que identifica los flujos de comercio entre el Brasil y Chile.

BRAMEX $_{i j}=$ Es la variable dicotómica que identifica los flujos de comercio entre el Brasil y México.

BRAURY $_{i j}=$ es la variable ficticia que identifica los flujos de comercio entre el Brasil y el Uruguay.

$\mathrm{ARGBRA}_{i}=$ es la variable dicotómica que identifica todas las otras importaciones de la Argentina o del Brasil (excluidas las que ya fueron consideradas por las variables aludidas anteriormente, por ejemplo, provenientes del Brasil, la Argentina, Chile, México y Uruguay).

$\mathrm{ARG}_{j}=$ es la variable dicotómica que identifica otras exportaciones de la Argentina (sin incluir las dirigidas al Brasil, Chile, México y el Uruguay).

$\mathrm{BRA}_{j}=$ es la variable dicotómica que identifica otras exportaciones del Brasil (sin incluir las dirigidas a la Argentina, Chile, México y el Uruguay).

La hipótesis 3.1 será verdadera si hay un incremento significativo de las exportaciones argentinas, brasileñas o ambas a cualquier mercado extrabloque $\left(\mathrm{ARG}_{j}\right.$,
ARGCHL $_{i j}$, ARGURY $_{i j}$, ARGMEX $_{i j}$, BRA $_{j}$, BRACHL $_{i j}$, BRAURY $_{i j}$, BRAMEX $i j$ ) entre los períodos $2^{\circ}$ y $3^{\circ}$.

La hipótesis 3.2 será verdadera si hay una merma del comercio intrabloque $\left(\right.$ ARGBRA $_{i j}$ ) de la misma magnitud que el incremento conjunto de las exportaciones a todos los demás mercados entre los períodos $2^{\circ}$ y $3^{\circ}$.

En la estimación de los modelos de gravedad 1 y 2 , la variable dependiente fue siempre el logaritmo natural del valor constante de las importaciones, y las variables independientes fueron las mencionadas en la ecuación (3). Como se señaló anteriormente, la diferencia entre los modelos 1 y 2 surge de la diferente desagregación de los mercados de exportación para la Argentina y el Brasil. En el modelo 1 se trata a la Argentina y el Brasil como bloque y se toman en cuenta tanto su comercio intrabloque $\left(\right.$ ARGBRA $_{i j}$ ) como las importaciones desde fuera del bloque $\left(\mathrm{ARGBRA}_{\mathrm{i}}\right.$ ) y las exportaciones al exterior del

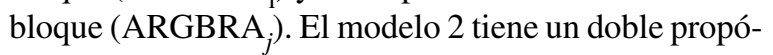
sito. En primer lugar, se intenta identificar diferencias entre los patrones de exportación de la Argentina y el Brasil y, por lo tanto, se incluyen variables dicotómicas separadas para cada uno de estos países (en lugar de tratarlos como bloque, como se hizo en el modelo 1). En segundo lugar, se desagregan las exportaciones a socios con acuerdos preferenciales de comercio, mientras que los socios sin acuerdos preferenciales de comercio se consideran en conjunto como exportaciones fuera de ese tipo de acuerdos.

La interpretación de la categoría de base (el término constante) es la misma en ambos modelos. Representa el comercio bilateral mundial que ocurre independientemente del desempeño de variables incluidas en los modelos. Como no hay diferencia en las variables de control incluidas en los modelos 1 y 2 (en el último la variable dicotómica $\mathrm{ARGBRA}_{j}$ del modelo 1 se divide en 8 nuevas variables: $\mathrm{ARG}_{j}, B R A_{j}, \mathrm{ARGURY}_{i j}, \mathrm{ARGCHL}_{i j}$, ARGMEX $_{i j}$, BRAURY $_{i j}$, BRACHL $_{i j}$, BRAMEX $_{i j}$ ), ninguno de los coeficientes de todas las demás variables (el término constante incluido) debería diferir drásticamente de las estimaciones de los modelos 1 y 2.

\section{Métodos de estimación}

Existen diferentes métodos de estimación alternativos que podrían usarse para estimar modelos de gravedad utilizando datos en panel de países. El panel empleado en este estudio incluye un máximo de 78 países exportadores y 103 países importadores de todo el mundo, y abarca el período 1991-2005. Dado que los flujos de comercio se refieren a un único sector, podría haber muchos pares bilaterales (país importador - país exportador) en que 
no existan flujos comerciales en algún período. Esta característica de base de datos censurada conduce a la elección de un modelo Tobit como primera opción de método de estimación.

Sin embargo, la información faltante en el conjunto de datos podría deberse tanto a falta de información (no se informa cuando no se comerció nada) como a ausencia de relación bilateral entre pares de países. Para aumentar la robustez del estudio y evitar imputar valores cero a todos los datos faltantes, se mantuvieron como parte de la base de datos solo las relaciones bilaterales en que existía un flujo bilateral por lo menos en tres años entre 1989 y 2006. Cuando el dato era faltante implicaba que no había habido comercio en ese período y por tanto se imputaba un cero a su flujo comercial. Para esos casos se asumió que la relación bilateral existía y que la entrada de los datos faltantes era cero. En los demás casos, se asumió que la relación comercial no existía y se los dejó fuera del análisis. Sin embargo, como se mencionó, la cobertura no se distribuyó de la misma manera a lo largo del tiempo. En particular, en el período anterior a 1994 hubo muchos países que no informaron ni importaciones ni exportaciones. De esta forma, el imputar cero a los valores faltantes podría implicar generar un sesgo en la relación, al ingresar flujos cero en esos primeros años en que pudo haber flujos positivos sin informar.

Un procedimiento básico de remuestreo (bootstrapping) de datos para períodos y flujos bilaterales mostró resultados inconsistentes para estimaciones de panel Tobit. Solo se logró la consistencia cuando se excluyó el período previo al Mercosur (es decir, cuando se consideraron datos desde 1995 en adelante). Dada la pregunta de investigación planteada, no era una alternativa centrar el análisis en el período 1995-2005.

Por consiguiente, la segunda mejor alternativa fue mantener solamente flujos positivos para el análisis, evitando de este modo la imputación de ceros. De esta forma, se hizo una estimación con mínimos cuadrados ordinarios (MCO) para el valor medio en los años comprendidos en los tres períodos diferentes definidos en la subsección 3 de la sección V.

\section{VI}

\section{Resultados empíricos}

\section{Robustez de la estimación, bondad de ajuste y variables del modelo de gravedad}

En los cuadros 2 y 3 se presentan los resultados de los modelos 1 y 2 , respectivamente. Como se señaló, estos dos modelos incluyen una cantidad diferente de variables dicotómicas que representan el comercio extrabloque y, por lo tanto, los coeficientes para variables dicotómicas relativas a mercados de exportación de la Argentina o el Brasil (con el subíndice $j$ ) difieren entre estos dos modelos. Empero, ninguna de las demás variables incluidas en los modelos 1 y 2 cambian significativamente. Esto indicaría qué diseño de la investigación es adecuado, lo que a su vez asegura la robustez de la estimación.

Además, la bondad de ajuste de modelos estimados para diferentes períodos es razonable, ubicándose $\mathrm{R}^{2}$ en el rango del $39 \%$ al $51 \%$.

Empezando el análisis por las variables típicas de los modelos de gravedad, se observa que la mayoría de ellas son generalmente significativas y muestran los signos correctos. El tamaño y la distancia son las variables clave de los modelos de gravedad. En este análisis se emplean tres variables dicotómicas para el tamaño y seis para la distancia. En general, los resultados son los esperados: el tamaño afecta positivamente al comercio y la distancia lo hace negativamente. También se extendió el modelo de gravedad a fin de incluir una variable dicotómica para la competitividad de la industria automotriz del importador en relación con la del exportador $\left(\mathrm{RCA}_{i j}\right)$ y diversas variables dicotómicas para herramientas de regulación (bloques comerciales) que, se podría decir, acortan la distancia entre socios. A continuación se examinan los resultados para este grupo de variables:

\section{Tamaño}

- Cuando el tamaño del mercado del importador se representa por el PIB hay un efecto positivo y marcado en el comercio. Este efecto parece haber aumentado con el tiempo. El tamaño del mercado también se representó por la población del país importador; sin embargo, en ese caso se encontraron resultados opuestos, aunque mucho más débiles (los países con mayor población importan menos automóviles). Esto puede estar relacionado con el 
bajo poder adquisitivo de los países superpoblados que, en general, no pueden costear la compra de automóviles, que son bienes con una alta elasticidad ingreso de la demanda (bienes de lujo). El tamaño del territorio del importador solo es significativo (y positivo, aunque débil) en el último período (2001-2005) aquí analizado.

- Desde el punto de vista del exportador, el tamaño se representa por la capacidad de producción que, tal como se esperaba, tiene un efecto positivo, fuerte y significativo en el comercio. De manera similar, la población del país exportador, otra variable dicotómica del tamaño del exportador, también muestra un efecto significativo y positivo —aunque más débil- en el comercio. Sin embargo, si se mide el tamaño del exportador en términos de superficie del territorio, se observa el resultado opuesto: los exportadores más pequeños comercian más. Este resultado aparentemente anómalo es esperable dado que los productores de automóviles residen en mayor medida en países pequeños de Europa y Asia. De hecho, en 2005 el $60 \%$ de los países productores, que representaban el $53 \%$ de la producción mundial (sin incluir la producción de China y la India), se ubicaban en Asia y Europa.

\section{Distancia}

- Las principales variables para representar la distancia geográfica son el alejamiento y la distancia en kilómetros entre socios. Esta última es significativa y muestra un coeficiente alto con el signo correcto; cuando la distancia entre socios se acrecienta un $1 \%$, el comercio también se reduce un $1 \%$. Este efecto parece haber aumentado con el tiempo. El alejamiento (distancia media de todos los socios) tiene el efecto opuesto al esperado: los países tienden a importar automóviles de socios remotos. Esto puede deberse a que los Estados Unidos y el Japón, dos países bastante remotos para el resto del mundo, muestran un elevado grado de diversificación del mercado y un peso importante en las exportaciones mundiales. ${ }^{14}$ La variable dicotómica para la distancia cultural fue el idioma. Compartir un mismo idioma se vuelve un factor importante para explicar el comercio solo durante el último

\footnotetext{
14 Además, este coeficiente cambia de signo si se incluyen valores cero en los modelos Tobit. Por lo tanto, parece que la lejanía afecta negativamente a la creación de nuevas relaciones bilaterales, pero no necesariamente a la intensificación del comercio entre los socios existentes.
}

período (con excepción de los países donde se habla árabe, probablemente debido a su muy escasa participación en la producción de automóviles).

\section{Ventaja Comparativa Manifiesta}

- Estas variables muestran los signos negativos esperados; cuanto más competitivo es el importador con relación al exportador, menor es el nivel de comercio entre ellos.

\section{Bloques}

- la mayoría de las variables dicotómicas significativas muestran los signos esperados, lo que indica que las regulaciones de los acuerdos comerciales han contribuido a acortar las distancias. ${ }^{15}$

\section{Evidencia que respalda las hipótesis principales del estudio}

Como puede verse en el cuadro 2, la Argentina y el Brasil comerciaron entre sí más de lo esperado según la versión ampliada del modelo de gravedad (ARGBRA es significativo y positivo en todos los períodos). Más precisamente, en el período 1991-1994, la Argentina y el Brasil comerciaron 34 veces más de lo esperado de acuerdo al modelo de gravedad. ${ }^{16}$ Sin embargo, en la segunda etapa ("hacia la integración"), después de que estos dos países firmaran su acuerdo comercial en diciembre de 1994, hubo 147 veces más comercio entre ellos de lo esperado según el modelo de gravedad. En otras palabras, el acuerdo parece haber tenido un efecto muy importante en el comercio intrabloque. ${ }^{17}$ En la tercera etapa ("profundización de la integración") el comercio intrabloque se redujo con relación al período anterior; aun así, la Argentina y el Brasil comerciaron 65 veces más de lo esperado de acuerdo con el modelo de gravedad.

\footnotetext{
${ }^{15}$ La única excepción es el bloque de los países de la ASEAN, que parecen comerciar menos entre sí que lo que predice el modelo de gravedad, sobre todo en los primeros dos períodos. Este resultado puede deberse a dos factores. En primer lugar, en esos años el déficit comercial del bloque superó el $100 \%$ (en forma conjunta los países importaron más del doble de lo que exportaron), por lo tanto, la mayoría de las importaciones provinieron de fuera del bloque. En segundo lugar, los principales productores de la ASEAN (Indonesia, Malasia y Tailandia) exportan sobre todo a destinos fuera del bloque.

${ }^{16}$ Como el modelo se estimó en logaritmos, el efecto de la variable dicotómica resulta de la fórmula: $=(\exp ($ coeficiente de variable dicotómica)-1)*100 (si se expresa en porcentajes). En el caso mencionado, $=(\exp (3,56)-1)=34$.

${ }^{17} \mathrm{La}$ diferencia en el coeficiente para $\mathrm{ARGBRA}_{i j}$ entre los períodos $1^{\circ}$ y $2^{\circ}$ es significativa (valor $\mathrm{p} 0,06$ ).
} 
CUADRO 2

\section{Comercio intrabloque: regresión de mínimos cuadrados ordinarios para el modelo de gravedad 1 estimada para diferentes períodos, 1991-1994, 1995-2000 y 2001-2005}

\begin{tabular}{|c|c|c|c|c|c|c|c|c|c|}
\hline \multirow{3}{*}{ Variable } & \multicolumn{9}{|c|}{ Modelo 1} \\
\hline & \multicolumn{3}{|c|}{$\begin{array}{c}\text { Etapa de "no integración" } \\
\text { 1991-1994 }\end{array}$} & \multicolumn{3}{|c|}{$\begin{array}{l}\text { Etapa "hacia la integración" } \\
\text { 1995-2000 }\end{array}$} & \multicolumn{3}{|c|}{$\begin{array}{l}\text { Etapa de "profundización de la integración" } \\
\text { 2001-2005 }\end{array}$} \\
\hline & Coeficiente & Valor $\mathrm{p}$ & Significación & Coeficiente & Valor $\mathrm{p}$ & Significación & Coeficiente & Valor $\mathrm{p}$ & Significación \\
\hline $\ln Y_{-} i$ & 0,36 & 0,00 & $* * *$ & 0,40 & 0,00 & $* * *$ & 0,50 & 0,00 & $* * *$ \\
\hline $\ln \overline{P C}_{-} j$ & 0,58 & 0,00 & $* * *$ & 0,63 & 0,00 & $* * *$ & 0,89 & 0,00 & $* * *$ \\
\hline $\ln N_{i} i$ & $-0,06$ & 0,30 & & $-0,09$ & 0,04 & $* *$ & $-0,11$ & 0,01 & $*$ \\
\hline $\ln N_{-} j$ & 0,40 & 0,00 & $* * *$ & 0,41 & 0,00 & $* * *$ & 0,22 & 0,00 & $* * *$ \\
\hline $\ln T_{-} i$ & 0,00 & 0,94 & & 0,01 & 0,68 & & 0,08 & 0,01 & $* *$ \\
\hline $\ln \mathrm{T}_{j} j$ & $-0,42$ & 0,00 & $* * *$ & $-0,41$ & 0,00 & $* * *$ & $-0,33$ & 0,00 & $* * *$ \\
\hline ln $\overline{A D} \_i j$ & 0,52 & 0,00 & $* *$ & 0,44 & 0,00 & $* * *$ & 0,26 & 0,04 & $*$ \\
\hline lnD_ij & $-0,90$ & 0,00 & $* * *$ & $-0,93$ & 0,00 & $* * *$ & $-1,02$ & 0,00 & $* * *$ \\
\hline $\mathrm{A} \_i \bar{j}$ & 1,21 & 0,00 & $* * *$ & 0,67 & 0,01 & $* *$ & 0,74 & 0,00 & $* *$ \\
\hline I_ $i$ & $-0,18$ & 0,22 & & 0,07 & 0,53 & & $-0,01$ & 0,93 & \\
\hline$I_{-} j$ & 0,88 & 0,00 & $* * *$ & 0,74 & 0,00 & $* * *$ & 0,94 & 0,00 & $* * *$ \\
\hline $\overrightarrow{\mathrm{LL}_{-} i} i$ & $-0,08$ & 0,59 & & $-0,30$ & 0,01 & $*$ & $-0,35$ & 0,00 & $* *$ \\
\hline $\mathrm{LL}_{-} j$ & $-0,27$ & 0,18 & & $-0,31$ & 0,03 & $*$ & $-0,25$ & 0,11 & \\
\hline $\mathrm{LSp} \_i j$ & $-0,07$ & 0,76 & & 0,23 & 0,24 & & 0,47 & 0,02 & $*$ \\
\hline LEn_ij & 0,24 & 0,24 & & 0,41 & 0,01 & $*$ & 0,42 & 0,02 & $*$ \\
\hline LAr_ij & $-1,89$ & 0,04 & $*$ & $-1,92$ & 0,00 & $* *$ & $-0,90$ & 0,17 & \\
\hline LFr_ij & 0,97 & 0,05 & $\dagger$ & 1,05 & 0,00 & $* *$ & 1,88 & 0,00 & $* * *$ \\
\hline LOt_ij & 2,28 & 0,00 & $* * *$ & 1,78 & 0,00 & $* *$ & 1,46 & 0,01 & $* *$ \\
\hline bloc_andean_ij & 0,20 & 0,80 & & 1,46 & 0,04 & $*$ & 1,20 & 0,09 & $\dagger$ \\
\hline bloc_asean_ij & $-2,09$ & 0,00 & $* *$ & $-0,89$ & 0,05 & $\dagger$ & $-0,69$ & 0,10 & \\
\hline bloc_cacm_ij & 0,86 & 0,21 & & 1,94 & 0,00 & $* *$ & 1,95 & 0,00 & $* *$ \\
\hline bloc_caricom_ij & $-0,73$ & 0,21 & & $-0,17$ & 0,92 & & (disminuye) & & \\
\hline bloc_cemac_ij & $-0,16$ & 0,96 & & $-1,37$ & 0,33 & & $-0,22$ & 0,88 & \\
\hline bloc_comesa_ij & $-0,38$ & 0,53 & & $-0,14$ & 0,78 & & $-0,02$ & 0,97 & \\
\hline bloc_eccas_ij & $-2,36$ & 0,36 & & (disminuye) & & & (disminuye) & & \\
\hline bloc_ecowas_ $i j$ & $-2,17$ & 0,23 & & $-0,75$ & 0,09 & $\dagger$ & 0,86 & 0,14 & \\
\hline bloc_efta_ $\overline{\mathrm{i}} \overline{\mathrm{j}}$ & 1,73 & 0,24 & & 1,15 & 0,23 & & 1,49 & 0,13 & \\
\hline bloc_eu25_ $\overline{i j}^{\mathrm{J}}$ & 2,89 & 0,00 & $* * *$ & 2,89 & 0,00 & $* * *$ & 2,22 & 0,00 & $* * *$ \\
\hline bloc_gcc_ij & (disminuye) & & & (disminuye) & & & (disminuye) & & \\
\hline bloc_nafta_ $i j$ & 5,12 & 0,00 & $* * *$ & 6,13 & 0,00 & $* * *$ & 4,93 & 0,00 & $* * *$ \\
\hline bloc_sadc_ $i j$ & 1,87 & 0,01 & $* *$ & 1,39 & 0,00 & $* * *$ & 1,83 & 0,00 & $* * *$ \\
\hline bloc_saarc_ $i j$ & $-2,37$ & 0,01 & $* *$ & $-1,08$ & 0,12 & & $-0,74$ & 0,33 & \\
\hline bloc_waemu_ $i j$ & $-2,70$ & 0,39 & & $-0,06$ & 0,94 & & 0,55 & 0,54 & \\
\hline RCA_ij & $-0,0003$ & 0,00 & $* * *$ & $-0,0012$ & 0,00 & $* * *$ & $-0,0003$ & 0,00 & $* *$ \\
\hline ARGBRA_ $i$ & 0,39 & 0,25 & & 0,49 & 0,08 & $\dagger$ & $-0,30$ & 0,31 & \\
\hline ARGBRA_j & 0,06 & 0,81 & & 0,17 & 0,43 & & 0,55 & 0,01 & $* *$ \\
\hline ARGBRA_ $i j$ & 3,56 & 0,05 & $\dagger$ & 5,00 & 0,00 & $* *$ & 4,19 & 0,01 & $*$ \\
\hline constante & $-11,21$ & 0,00 & $* * *$ & $-12,72$ & 0,00 & $* * *$ & $-17,51$ & 0,00 & $* * *$ \\
\hline $\mathrm{N}$ & 3013 & & & 4019 & & & 3752 & & \\
\hline Ajuste $R^{2}$ & 0,39 & & & 0,47 & & & 0,51 & & \\
\hline
\end{tabular}

Fuente: elaboración propia sobre la base de diferentes fuentes.

$\dagger$ si $\mathrm{p}<0,10 ; *$ si $\mathrm{p}<0,05 ; * *$ si $\mathrm{p}<0,01 ; * * *$ si $\mathrm{p}<0,001$

Notas: Andean: Comunidad Andina. Asean: Asociación de Naciones del Asia Sudoriental. Cacm: Mercado Común Centroamericano (mccA). Caricom: Comunidad del Caribe. Caemc: Comunidad Económica y Monetaria del África Central (CEMAC). Comesa: Mercado Común para el África Oriental y Meridional. Eccas: Comunidad Económica de los Estados de África Central (CEEAC). Ecowas: Comunidad Económica de los Estados de África Occidental (CEDEAO). Efta: Asociación Europea de Libre Comercio (AELc). EU: Unión Europea. Gcc: Consejo de Cooperación del Golfo (GCC). Nafta: Tratado de Libre Comercio de América del Norte (TLC). Sadc: Comunidad de África Meridional para el Desarrollo (sADC). Saarc: Asociación del Asia Meridional para la Cooperación Regional (SAARC). Waemu: Unión Económica y Monetaria del África Occidental (UEMAO). RCA: ventaja comparativa manifiesta. ARGBRA: Argentina/Brasil. Valor p: valor de probabilidad. 
En el gráfico 9 se aprecia la evolución de los coeficientes del comercio intrabloque entre la Argentina y el Brasil cuando las ecuaciones del modelo de gravedad se estiman por año. Como puede verse, el comercio entre estos dos países se expandió desde comienzos de la década de 1990; sin embargo, no fue sino hasta después del primer acuerdo (a fines de 1994) que la Argentina y el Brasil comerciaron un volumen considerablemente mayor de lo esperado de acuerdo con la ecuación de gravedad. Este efecto fue el más pronunciado en el período 1996-1998. En 1999 la recesión brasileña podría haber desviado las exportaciones de la Argentina a otros destinos; luego se observa una caída del comercio intrabloque en la época de la crisis argentina de 2002. Estos factores macroeconómicos pueden explicar por qué el segundo acuerdo suscrito el año 2000, cuando la integración entre la Argentina y el Brasil se profundizó, no incrementó el comercio intrabloque como cabría esperar.

Por lo tanto, sobre la base de esta evidencia podría rechazarse la hipótesis 2 pero no la hipótesis 1 . Sin embargo, para concluir que hubo creación de comercio después del acuerdo de 1994 es necesario determinar si el aumento del coeficiente de comercio intrabloque compensa la disminución del coeficiente de comercio extrabloque, medido por la variable ARGBRA $i$. En el cuadro 2 se muestra que no hubo disminución en el comercio extrabloque después de 1994 y que, por lo tanto, no puede rechazarse la hipótesis 1. En otras palabras, después del primer acuerdo de 1994, se creó comercio para la Argentina y el Brasil. Esto no ocurrió tras el acuerdo firmado en el año 2000 (es decir, la evidencia respalda la hipótesis 1 , pero no la hipótesis 2).
Las exportaciones a otros destinos durante la "profundización de la integración" (etapa 3) fueron un $\left.73 \%(=\exp (0,55)-1)^{*} 100\right)$ más elevadas de lo esperado de acuerdo con el modelo de gravedad. En las etapas 1 y 2 estos países exportaron a otros destinos tan solo valores similares a aquellos esperados por el modelo de gravedad (es decir, los coeficientes de ARGBRA $_{j}$ no fueron significativos). La significatividad del coeficiente ARGBRA $_{j}$ en la etapa 3 parece indicar que los mercados extrabloque fueron particularmente importantes para las exportaciones de la Argentina y el Brasil durante la etapa de "profundización de la integración". Sin embargo, la diferencia entre dicho coeficiente y los de las etapas 1 o 2 no es significativa. Aunque esto puede explicarse estadísticamente por el amplio desvío estándar de los coeficientes en los períodos $1^{\circ}$ y $2^{\circ}$, no puede afirmarse categóricamente que la Argentina, el Brasil o ambos incrementaron la diversificación del mercado de exportación después del acuerdo del año 2000 (ya sea como consecuencia del cambio de régimen que dio inicio a la etapa de "profundización de la integración" o debido al desorden macroeconómico). Por lo tanto, aunque en este período los mercados extrabloque alcanzaron una importancia considerable, la evidencia disponible conduce a rechazar las hipótesis 3.1 y 3.2 cuando la Argentina y el Brasil se consideran como un único bloque.

La información que se presenta en el cuadro 3 ayuda a distinguir las estrategias de exportación seguidas separadamente por la Argentina y el Brasil y, por ende, ello permite someter a prueba las hipótesis 3.1 y 3.2 para cada país. En este cuadro 3 se aprecian los resultados de las estimaciones del modelo 2, prestando especial

GRÁFICO 9

Coeficientes para variables dicotómicas ARGBRA ${ }_{-i j}$ en estimaciones de mínimos cuadrados ordinarios de la ecuación de gravedad por año, 1990-2005

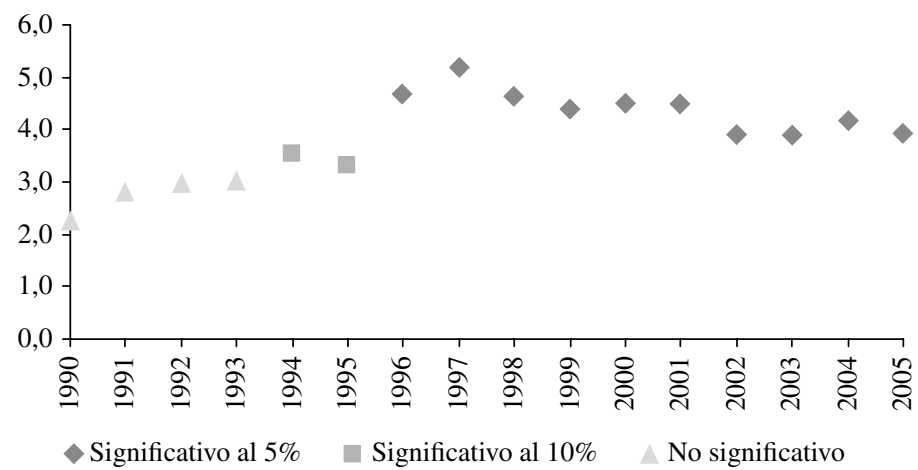

Fuente: elaboración propia sobre la base de diferentes fuentes (véase la sección V, número 2). 
CUADRO 3

Diversificación de mercados: regresión de mínimos cuadrados ordinarios para el modelo de gravedad 2 estimada para diferentes períodos,1991-1994, 1995-2000 y 2001-2005

\begin{tabular}{|c|c|c|c|c|c|c|c|c|c|}
\hline \multicolumn{10}{|c|}{ Modelo 2} \\
\hline \multirow{2}{*}{ Variable } & \multicolumn{3}{|c|}{$\begin{array}{c}\text { Etapa de "no integración" } \\
\text { 1991-1994 }\end{array}$} & \multicolumn{3}{|c|}{$\begin{array}{c}\text { Etapa "hacia la integración" } \\
1995-2000\end{array}$} & \multicolumn{3}{|c|}{$\begin{array}{l}\text { Etapa de "profundización de la integración" } \\
\text { 2001-2005 }\end{array}$} \\
\hline & Coeficiente & Valor $\mathrm{p}$ & Significación & Coeficiente & Valor $\mathrm{p}$ & Significación & Coeficiente & Valor $\mathrm{p}$ & Significación \\
\hline $\ln Y_{-} i$ & 0,36 & 0,00 & $* * *$ & 0,40 & 0,00 & $* * *$ & 0,50 & 0,00 & $* * *$ \\
\hline $\operatorname{lnP} \bar{C}_{-} j$ & 0,58 & 0,00 & $* * *$ & 0,63 & 0,00 & $* * *$ & 0,89 & 0,00 & $* * *$ \\
\hline $\ln N_{-} \vec{i}$ & $-0,05$ & 0,35 & & $-0,09$ & 0,04 & $* *$ & $-0,11$ & 0,01 & $* *$ \\
\hline $\ln N_{-} j$ & 0,40 & 0,00 & $* * *$ & 0,41 & 0,00 & $* * *$ & 0,21 & 0,00 & $* * *$ \\
\hline $\ln \mathrm{T}_{-} i$ & $-0,01$ & 0,81 & & 0,01 & 0,75 & & 0,08 & 0,01 & $* * *$ \\
\hline $\ln \mathrm{T}_{-j} j$ & $-0,42$ & 0,00 & $* * *$ & $-0,41$ & 0,00 & $* * *$ & $-0,33$ & 0,00 & $* * *$ \\
\hline $\ln \mathrm{AD} \_i j$ & 0,47 & 0,00 & $* * *$ & 0,41 & 0,00 & $* * *$ & 0,24 & 0,06 & $*$ \\
\hline lnD_ij & $-0,87$ & 0,00 & $* * *$ & $-0,91$ & 0,00 & $* * *$ & $-1,01$ & 0,00 & $* * *$ \\
\hline $\mathrm{A} \_i \bar{j}$ & 1,08 & 0,00 & $* * *$ & 0,56 & 0,02 & $* *$ & 0,70 & 0,01 & $* * *$ \\
\hline I_ $i$ & $-0,17$ & 0,23 & & 0,08 & 0,51 & & $-0,01$ & 0,95 & \\
\hline $\mathrm{I}_{-}^{-} j$ & 0,87 & 0,00 & $* * *$ & 0,74 & 0,00 & $* * *$ & 0,93 & 0,00 & $* * *$ \\
\hline $\overrightarrow{\mathrm{LL}} \_i$ & $-0,07$ & 0,65 & & $-0,28$ & 0,01 & $* *$ & $-0,35$ & 0,00 & $* * *$ \\
\hline $\mathrm{LL}_{-} j$ & $-0,27$ & 0,19 & & $-0,31$ & 0,03 & $* *$ & $-0,25$ & 0,11 & \\
\hline $\mathrm{LSp} \_i j$ & 0,05 & 0,84 & & 0,29 & 0,15 & & 0,47 & 0,03 & $* *$ \\
\hline LEn_ $i j$ & 0,25 & 0,22 & & 0,41 & 0,01 & $* *$ & 0,42 & 0,02 & $* *$ \\
\hline LAr_ $i j$ & $-1,82$ & 0,04 & $* *$ & $-1,88$ & 0,00 & $* * *$ & $-0,89$ & 0,18 & \\
\hline LFr_ij & 0,98 & 0,05 & $* *$ & 1,06 & 0,00 & $* * *$ & 1,88 & 0,00 & $* * *$ \\
\hline LOt_ij & 2,28 & 0,00 & $* * *$ & 1,76 & 0,00 & $* * *$ & 1,47 & 0,01 & $* * *$ \\
\hline bloc_andean_ $i j$ & 0,21 & 0,79 & & 1,50 & 0,03 & $* *$ & 1,24 & 0,09 & $*$ \\
\hline bloc_asean_i $\overline{i j}$ & $-1,99$ & 0,00 & $* * *$ & $-0,83$ & 0,07 & $*$ & $-0,66$ & 0,12 & \\
\hline bloc_cacm_ij & 0,84 & 0,22 & & 1,96 & 0,00 & $* * *$ & 1,98 & 0,00 & $* * *$ \\
\hline bloc_caricom_ij & $-0,73$ & 0,68 & & $-0,17$ & 0,92 & & (disminuye) & & \\
\hline bloc_cemac_i $\overline{i j}$ & $-0,02$ & 0,99 & & $-1,22$ & 0,39 & & $-0,17$ & 0,91 & \\
\hline bloc_comesa_ij & $-0,33$ & 0,58 & & $-0,12$ & 0,81 & & $-0,01$ & 0,98 & \\
\hline bloc_eccas_ij & $-2,29$ & 0,37 & & (disminuye) & & & (disminuye) & & \\
\hline bloc_ecowas_ij & $-2,00$ & 0,27 & & $-0,68$ & 0,12 & & 0,89 & 0,13 & \\
\hline bloc_efta_ij & 1,76 & 0,23 & & 1,15 & 0,23 & & 1,48 & 0,13 & \\
\hline $\begin{array}{l}\text { bloc_eu } 25 \_i j \\
\text { bloc_occ } i j\end{array}$ & $\begin{array}{c}2,92 \\
\text { (disminuve) }\end{array}$ & 0,00 & $* * *$ & $\begin{array}{c}2,91 \\
\text { (disminuve) }\end{array}$ & 0,00 & $* * *$ & 2,22 & 0,00 & $* * *$ \\
\hline $\begin{array}{l}\text { bloc_gcc_lj } \\
\text { bloc_nafta } i j\end{array}$ & $\begin{array}{c}\text { (disminuye) } \\
5,25\end{array}$ & 0,00 & $* * *$ & $\begin{array}{c}\text { (disminuye) } \\
6,23\end{array}$ & 0.00 & $* * *$ & $\begin{array}{c}\text { (disminuye) } \\
4.98\end{array}$ & 0,00 & $* * *$ \\
\hline bloc_sadc_ij & 1,97 & 0,01 & $* * *$ & 1,47 & 0,00 & $* * *$ & 1,86 & 0,00 & $* * *$ \\
\hline bloc_saarc_ij & $-2,27$ & 0,00 & $* * *$ & $-0,99$ & 0,15 & & $-0,70$ & 0,36 & \\
\hline bloc_waemu_ij & $-2,82$ & 0,37 & & $-0,08$ & 0,92 & & 0,54 & 0,54 & \\
\hline RCA_ij & $-0,0003$ & 0,00 & $* * *$ & $-0,0012$ & 0,00 & $* * *$ & $-0,0003$ & 0,00 & $* * *$ \\
\hline ARGBRA_ $i$ & 0,31 & 0,38 & & 0,40 & 0,17 & & $-0,39$ & 0,19 & \\
\hline $\mathrm{ARG}_{-} j$ & $-1,01$ & 0,02 & $* *$ & $-0,80$ & 0,02 & $* *$ & 0,12 & 0,74 & \\
\hline $\mathrm{BRA} j$ & 0,43 & 0,15 & & 0,59 & 0,03 & $* *$ & 0,60 & 0,03 & $* *$ \\
\hline ARGBRA_ $i j$ & 3,92 & 0,03 & $* *$ & 5,43 & 0,00 & $* * *$ & 4,91 & 0,00 & $* * *$ \\
\hline ARGURY_ij & 2,93 & 0,11 & & 1,76 & 0,29 & & 0,17 & 0,92 & \\
\hline ARGCHL_ij & 1,29 & 0,48 & & 1,73 & 0,30 & & 0,79 & 0,64 & \\
\hline ARGMEX_ $i j$ & $-2,05$ & 0,26 & & 1,18 & 0,48 & & 3,36 & 0,05 & $* *$ \\
\hline BRAURY_ij & 2,46 & 0,17 & & 4,08 & 0,01 & $* *$ & 2,12 & 0,21 & \\
\hline BRACHL_ $i j$ & 2,48 & 0,17 & & 1,39 & 0,40 & & 1,64 & 0,33 & \\
\hline BRAMEX_ij & 3,08 & 0,09 & $*$ & 3,92 & 0,02 & $* *$ & 4,27 & 0,01 & $* *$ \\
\hline Constante & $-11,03$ & 0,00 & $* * *$ & $-12,52$ & 0,00 & $* * *$ & $-17,34$ & 0,00 & $* * *$ \\
\hline $\mathrm{N}$ & 3013 & & & 4019 & & & 3752 & & \\
\hline Ajuste $\mathrm{R}^{2}$ & 0,39 & & & 0,48 & & & 0,51 & & \\
\hline
\end{tabular}

Fuente: elaboración propia sobre la base de diferentes fuentes.

$\dagger$ si $\mathrm{p}<0,10 ; *$ si $\mathrm{p}<0,05 ; * *$ si $\mathrm{p}<0,01 ; * * *$ si $\mathrm{p}<0,001$

Notas: Andean: Comunidad Andina. Asean: Asociación de Naciones del Asia Sudoriental. CACM: Mercado Común Centroamericano (MCCA). Caricom: Comunidad del Caribe. Caemc: Comunidad Económica y Monetaria del África Central (CEMAC). Comesa: Mercado Común para el África Oriental y Meridional. Eccas: Comunidad Económica de los Estados de África Central (CEEAC). Ecowas: Comunidad Económica de los Estados de África Occidental (CEDEAO). Efta: Asociación Europea de Libre Comercio (AELC). EU: Unión Europea. Gcc: Consejo de Cooperación del Golfo (GCC). Nafta: Tratado de Libre Comercio de América del Norte (TLC). Sadc: Comunidad de África Meridional para el Desarrollo (SADC). Saarc: Asociación del Asia Meridional para la Cooperación Regional (SAARC). Waemu: Unión Económica y Monetaria del África Occidental (UEMAO). RCA: ventaja comparativa manifiesta. ARGBRA: Argentina/Brasil. ARGURY: Argentina/Uruguay. ARGCHL: Argentina/Chile. ARgMEX: Argentina/México. BRA/URY: Brasil/Uruguay. BRACHL: Brasil/Chile. BRAMEX: Brasil/México. 
atención a los mercados que han sido promocionados por regulaciones comerciales recientes.

Entre los mercados con acuerdos preferenciales de comercio, México es el único que se ha transformado en un socio cada vez más importante para la Argentina y el Brasil. En primer lugar, las exportaciones de la Argentina a México fueron considerablemente diferentes de lo esperado según el modelo de gravedad en la etapa 3. En segundo lugar, las exportaciones del Brasil a México siempre fueron más elevadas de lo esperado por el modelo de gravedad y se incrementaron con el tiempo. Las diferencias en los coeficientes entre las diferentes etapas temporales no son significativas en ninguno de los dos países.

El comercio de Chile con la Argentina y el Brasil no fue mayor que el esperado de acuerdo con el modelo de gravedad. El del Uruguay fue mayor a lo predicho por el modelo de gravedad solo en el caso del Brasil después de firmar el acuerdo de 1994.

Con respecto a otros mercados de exportación para la Argentina y el Brasil, se observa que en las etapas 1 y 2 la Argentina exportó menos de lo esperado (según el modelo de gravedad) a otros mercados $\left(\mathrm{ARG}_{j}\right) \sin$ acuerdos comerciales preferenciales en concordancia con el modelo de gravedad. En la etapa 3, el coeficiente ya no fue negativo, pero tampoco significativo. El Brasil, en cambio, muestra coeficientes positivos y significativos para $\mathrm{BRA}_{j}$ en las etapas 2 y 3, lo que da cuenta de una mayor intensidad en sus estrategias de diversificación más allá de los mercados con acuerdos comerciales preferenciales. Además, un ejercicio similar a este, pero realizado sobre el ejercicio en el modelo 1 (por ejemplo, dividiendo la variable dicotómica $\mathrm{ARGBRA}_{j}$ en $\mathrm{ARG}_{j}$ $\mathrm{y} \mathrm{BRA}_{j}$ ) arroja los mismos resultados.

En suma, respecto de la hipótesis 3.1 (la diversificación de las exportaciones aumentó en la etapa 3) la evidencia parece indicar que:

i) La Argentina exportó a México más de lo esperado en ese período de acuerdo con el modelo de gravedad, no así en períodos anteriores.

ii) El Brasil siempre exportó a México más de lo esperado de acuerdo con el modelo de gravedad y este efecto tendió a aumentar con el paso del tiempo. iii) El Brasil exportó más de lo esperado (según el modelo de gravedad) a mercados sin acuerdos comerciales preferenciales en las etapas 2 y 3 . No obstante, las diferencias entre períodos no fueron significativas, por lo tanto estos efectos no pueden vincularse a un cambio en particular en el año 2000.

En suma, pareciera no haber evidencia de una mayor diversificación del comercio luego del año 2000 (ya sea debido a la firma de los acuerdos del año 2000 o a la recesión en el Mercosur). Por lo tanto, la hipótesis 3 - tanto en su forma 3.1 como en su forma 3.2 - también debe rechazarse cuando se analizan la Argentina y el Brasil por separado. La hipótesis 3.1 (por ejemplo, diversificación a nuevos mercados después del acuerdo del año 2000) se rechaza porque no aumentaron sistemáticamente las exportaciones a mercados extrabloque en la etapa 3 con relación a la etapa 2. La hipótesis 3.2 (por ejemplo, la diversificación sucedió a costa del comercio intrabloque) se rechaza utilizando la misma evidencia que para la hipótesis 3.1 como por el hecho de que el comercio intrabloque $\left(\right.$ ARGBRA $\left._{i j}\right)$ no cambió significativamente entre las etapas 2 y 3 .

Por último, aunque las hipótesis 3.1 y 3.2 se han rechazado, existe cierta evidencia que muestra la creciente importancia de México respecto del comercio intrabloque. En la etapa 2 el comercio intrabloque fue considerablemente más intenso que el efectuado entre la Argentina o el Brasil con cualquier otro socio con acuerdo comercial preferencial (incluido México). Sin embargo, en la etapa 3, si bien los resultados del comercio intrabloque superaron los de cualquier otra asociación, el coeficiente intrabloque no es muy diferente del coeficiente de comercio entre México y la Argentina o entre México y el Brasil. En otras palabras, México pareció incrementar su relevancia relativa (respecto del comercio intrabloque) como mercado de exportación para la Argentina y también para el Brasil. Este no es el caso del Uruguay, un socio que disminuyó su importancia en términos relativos, ni de Chile, cuya importancia relativa fue más o menos estable en el tiempo. 


\section{VII}

\section{Conclusiones}

La tendencia a la internacionalización de la industria automotriz mundial se ha profundizado desde los años noventa e incluye estrategias globales y regionales. Se ha dicho que las estrategias regionales son más eficientes porque balancean mejor las necesidades de alcanzar una escala eficiente con las de lograr diferenciación del producto.

El Mercosur ha sido una localización histórica para la producción de automóviles. Por ejemplo, algunas subsidiarias de empresas multinacionales comenzaron a producir en la región antes de hacerlo en lugares más desarrollados. Actualmente, el Mercosur es un área relevante tanto en términos de su participación mundial en producción como en exportaciones.

Sin embargo, aún no se ha logrado la plena integración en la región para la industria. La razón principal para la falta de un acuerdo pleno en la industria automotriz es que los miembros del Mercosur no han logrado un consenso respecto del arancel externo común (la Argentina y el Brasil prefieren aranceles elevados, mientras que el Uruguay y el Paraguay prefieren aranceles más bajos).

Actualmente, este es uno de los pocos sectores en que el comercio argentino/brasileño se administra según una serie de acuerdos con diferentes grados de intervención. El primero de esos acuerdos se logró en 1994 e inició la etapa que en este artículo se denomina "hacia la integración". En el segundo acuerdo de mayor compromiso con la integración regional, firmado en el año 2000, se estableció el comienzo de la etapa de "profundización de la integración". Sin embargo, en 2006 y nuevamente en 2008 se firmaron acuerdos que de alguna manera significaron un retroceso en la integración intrabloque (etapa de "retroceso de la integración"). El libre comercio intrabloque se pospuso hasta 2013 como consecuencia, sobre todo, de la preocupación de la Argentina con respecto a la competencia del Brasil.

Todos estos acuerdos tendieron a favorecer las estrategias de complementación dentro de las empresas multinacionales ubicadas en ambos países. En alguna medida, esto se logró durante la "etapa de profundización”, como se señala en Arza y López (2008b) al estudiar el caso argentino.

El propósito de este artículo fue analizar en qué medida el Mercosur (definido como la Argentina y el
Brasil solamente) se transformó en una plataforma de exportación de la producción automotriz. Más específicamente, el objetivo consistió en examinar si se creó comercio después de los acuerdos comerciales de fines de 1994 y del año 2000, y si ambos países diversificaron las exportaciones a otros mercados durante la etapa de "profundización de la integración" (2000-2005).

La metodología propuesta (la estimación de una ecuación de gravedad ampliada) requirió la construcción de una gran base de datos con información de diferentes fuentes. Una vez reunidas todas las variables necesarias para la estimación, se creó una base de datos no balanceada con un máximo de 78 países exportadores y 103 países importadores, con mediciones de los flujos comerciales en el período 1991-2005. Con el fin de cumplir el objetivo de la investigación, la muestra se dividió en tres períodos (antes y después de los acuerdos comerciales de 1994 y 2000) y se estimaron dos versiones diferentes del modelo de gravedad. La diferencia entre los modelos es que el segundo desagrega las exportaciones extrabloque de manera más amplia.

El análisis empírico arrojó la conclusión de que hubo creación de comercio genuino después del acuerdo de 1994. La Argentina y el Brasil comerciaron más entre sí de lo que cabía esperar según el modelo de gravedad. Además, el comercio intrabloque se incrementó significativamente después de 1994 (es decir, entre los períodos 1 y 2), sin perjuicio del comercio extrabloque. No obstante, posiblemente a consecuencia de la recesión del Brasil (y de la Argentina) de 1998-1999 y la crisis de la Argentina de 2001-2002, el acuerdo del año 2000 no se tradujo en un mayor aumento del comercio intrabloque.

La evidencia descriptiva muestra que tras la firma del segundo acuerdo en el año 2000 (durante la etapa de "profundización de la integración"), la región pareció exportar más que antes a otros destinos. Además, en el Brasil se aplicó una estrategia más agresiva de diversificación de las exportaciones, que fueron más allá de los mercados con acuerdos preferenciales de comercio. Esto puede relacionarse con que el bloque se estaba transformando en una plataforma de exportación después de que la Argentina y el Brasil profundizaran su acuerdo en el año 2000, o con que ambos países sufrieron desórdenes macroeconómicos en esos años y por lo tanto necesitaban buscar mercados extrabloque. 
En todo caso, aunque las estimaciones econométricas permiten ver que el comercio extrabloque (de la Argentina dirigido en su mayoría a México, pero del Brasil también a países sin acuerdos comerciales preferenciales) era mayor que el esperado por el modelo de gravedad y de hecho se incrementó en el período, ese aumento no fue estadísticamente significativo.

En suma, hubo evidencia de creación de comercio después del acuerdo de 1994, aunque no ocurrió lo mismo luego del acuerdo firmado en el año 2000. La creación de comercio se explica principalmente por el engrosamiento del comercio intrabloque. Si bien fue creciendo la participación en las exportaciones de países fuera del bloque, no existe evidencia suficiente para asegurar que la Argentina o el Brasil hayan logrado incrementar sistemáticamente su acceso a mercados extrabloque después de la firma de sus acuerdos de integración. En otras palabras, a juzgar por la evidencia recolectada hasta el año 2005, los acuerdos del Mercosur del año 2000 no lograron que la región se convirtiera todavía en una plataforma de exportación a otros mercados extrabloque.

ANEXO 1

Acuerdos de integración regionales seleccionados

\begin{tabular}{ll}
\hline Comunidad Andina: & Colombia, Ecuador, Estado Plurinacional de Bolivia y Perú. \\
\hline $\begin{array}{l}\text { ASEAn (Asociación de Naciones del Asia } \\
\text { Sudoriental): }\end{array}$ & $\begin{array}{l}\text { Brunei Darussalam, Camboya, Indonesia, Malasia, Myanmar, Filipinas, República Democrática } \\
\text { Popular Laos, Singapur, Tailandia y Vietnam. }\end{array}$ \\
\hline MCCA (Mercado Común Centroamericano): & Costa Rica, El Salvador, Guatemala, Honduras y Nicaragua. \\
\hline CARICOM (Comunidad del Caribe): & $\begin{array}{l}\text { Antigua y Barbuda, Bahamas, Barbados, Belice, Dominica, Granada, Guyana, Haití, Jamaica, } \\
\text { Montserrat, Saint Kitts y Nevis, Santa Lucía, San Vicente y las Granadinas, Suriname y } \\
\text { Trinidad y Tabago. }\end{array}$ \\
\hline
\end{tabular}

CEMAC (Comunidad Económica y Monetaria del Camerún, Chad, Congo, Guinea Ecuatorial, Gabón y República Centroafricana.

África Central):

COMESA (Mercado Común para el África Oriental Angola, Burundi, Comoras, Congo, Djibouti, Egipto, Eritrea, Etiopía, Jamahiriya Árabe Libia, y Meridional): Kenya, Madagascar, Malawi, Mauricio, Namibia, República Democrática del Congo, Rwanda, Seychelles, Sudán, Swazilandia, Uganda, Zambia y Zimbabwe.

CEEAC (Comunidad Económica de los Estados Angola, Burundi, Camerún, Chad, Congo, Gabón, Guinea Ecuatorial, República Centroafricana, de África Central): República Democrática del Congo, Rwanda y Santo Tomé y Príncipe.

CEDEAo (Comunidad Económica de los Estados Benin, Burkina Faso, Cabo Verde, Côte d'Ivoire, Gambia, Ghana, Guinea, Guinea-Bissau, de África Occidental): Liberia, Malí, Níger, Nigeria, Senegal, Sierra Leona y Togo.

AELC (Asociación Europea de Libre Comercio): $\quad$ Islandia, Liechtenstein, Noruega y Suiza.

UE 15 (Unión Europea (15 países)): $\quad$ Alemania, Austria, Bélgica, Dinamarca, España, Finlandia, Francia, Grecia, Irlanda, Italia, Luxemburgo, Países Bajos, Portugal, Reino Unido y Suecia.

UE 25 (Unión Europea (25 países)): Alemania, Austria, Bélgica, Chipre, Dinamarca, Estonia, Finlandia, Francia, Grecia, Hungría, Irlanda, Italia, Letonia, Lituania, Luxemburgo, Malta, Países Bajos, Polonia, Portugal, Eslovaquia, Eslovenia, España, Suecia, Reino Unido y República Checa.

GCC (Consejo de Cooperación del Golfo): $\quad$ Arabia Saudita, Bahrein, Emiratos Árabes Unidos, Kuwait, Omán, Qatar.

Mercosur (Mercado Común del Sur): Argentina, Brasil, Paraguay y Uruguay.

TLC (Tratado de Libre Comercio de América Canadá, México y Estados Unidos.

del Norte):

Acuerdo comercial preferencial de la Asociación $\quad$ Bangladesh, Bhután, India, Maldivas, Nepal, Pakistán y Sri Lanka.

del Asia Meridional para la Cooperación

Regional (SAARC):

SADC (Comunidad de África Meridional para Angola, Botswana, Lesotho, Madagascar, Malawi, Mauricio, Mozambique, Namibia, República el Desarrollo): Democrática del Congo, República Unida de Tanzania, Seychelles, Sudáfrica, Swazilandia, Zambia y Zimbabwe.

Uemao (Unión Económica y Monetaria del Benin, Burkina Faso, Côte d'Ivoire, Guinea-Bissau, Malí, Níger, Senegal y Togo. África Occidental):

Fuente: Selección de la autora sobre la base de datos estadísticos de la Organización Mundial del Comercio (OMC). 


\section{Bibliografía}

Aitken, N.D. (1973), "The effect of the Eec and Eeta on European Trade: a temporal cross-section analysis", American Economic Review, vol. 63, № 5, Nashville, Tennessee, American Economic Association.

Arza, V. y A. López (2008a), "Tendencias internacionales en la industria automotriz", La industria automotriz en el Mercosur, A. López (comp.), Montevideo, Red de Investigaciones Económicas del Mercosur.

(2008b), "El caso argentino", La industria automotriz en el Mercosur, A. López (comp.), Montevideo, Red de Investigaciones Económicas del Mercosur.

(2008c), "Introducción al libro: la visión regional", La industria automotriz en el Mercosur, A. López (comp.), Montevideo, Red de Investigaciones Económicas del Mercosur.

Bastos Tigre, P. y otros (1999), "Impacto del Mercosur en la dinámica del sector automotor", Impacto sectorial de la integración en el Mercosur, J.J. Taccone y L.J. Garay (comps.), Buenos Aires, Instituto para la Integración de América Latina y el Caribe (INTAL).

Bayoumi, T. y B. Eichengreen (1997), "Is regionalism simply a diversion? Evidence from the evolution of the Ec and Efta", Regionalism Versus Multilateral Trade Arrangements, T. Ito y A.O. Krueger (comps.), Chicago, University of Chicago Press.

Braga, C.A., R. Safadi y A. Yeats (1994), "Regional integration in the Americas: deja vu all over again?", World Economy, vol. 17, $\mathrm{N}^{\circ}$ 4, Oxford, Blackwell Publishing.

Cimoli, M. y J. Katz (2001), "Reformas estructurales, brechas tecnológicas y el pensamiento del Dr. Prebisch", documento presentado en el Seminario "El desarrollo en el siglo XXI", Santiago de Chile, Comisión Económica para América Latina y el Caribe (CEPAL).

Ciravegna, L. (2003), "Global and regional integration of production in the Mercosur automotive value chains, the case of Fiat", EADI Workshop: Clusters and Value Chains in the North and in the Third World, Novara, Università del Piemonte Orientale.

Filippini, C. y V. Molini (2003), "The determinants of East Asian trade flows: a gravity equation approach", Journal of Asian Economics, vol. 14, No 5, Amsterdam, Elsevier.

Frankel, J.A. (1997), Regional Trading Blocs in the World Economic System, Washington, D.C., Institute for International Economics.

Freyssenet, M. e Y. Lung (2000), "Between globalisation and regionalisation: what is the future of the motor industry", Global Strategies and Local Realities: The Auto Industry in Emerging Markets, J. Humphrey, Y. Lecler y M.S. Salerno (comps.), Nueva York, St. Martin's Press.
Humphrey, J. y A. Oeter (2000), "Motor industry policies in emerging markets: globalisation and the promotion of domestic industry", Global Strategies and Local Realities: The Auto Industry in Emerging Markets, J. Humphrey, Y. Lecler y M.S. Salerno (comps.), Nueva York, St. Martin's Press.

Humphrey, J., Y. Lecler y M.S. Salerno (2000), Global Strategies and Local Realities: The Auto Industry in Emerging Markets, J. Humphrey, Y. Lecler y M.S. Salerno (comps.), Nueva York, St. Martin's Press.

Humphrey, J. y O. Memedovic (2003), The Global Automotive Industry Value Chain: What Prospects for Upgrading by Developing Countries, Viena, Organización de las Naciones Unidas para el Desarrollo Industrial (ONUDI).

Krueger, A.O. (1999), "Trade creation and trade diversion under Nafta", NBER Working Paper, No. 7429, Cambridge, Massachusetts, National Bureau of Economic Research.

Laplane, M. y F. Sarti (2008), "O caso do Brasil", La industria automotriz en el Mercosur, A. López (comp.), Montevideo, Red de Investigaciones Económicas del Mercosur.

Motta Veiga, P. (2004), Foreign Direct Investment in Brazil: Regulation, Flows and Contribution to Development, Winnipeg, Instituto Internacional para el Desarrollo Sostenible.

Musila, J.W. (2005), "The intensity of trade creation and trade diversion in COMESA, ECCAS and ECOWAS: a comparative analysis", Journal of African Economies, vol. 14, N ${ }^{\circ}$ 1, Oxford, Oxford University Press.

Naciones Unidas (1986), Clasificación Industrial Internacional Uniforme de todas las actividades económicas, Rev. 3 (ST/ ESA/STAT/SER.M/34/Rev.3), Nueva York. Publicación de las Naciones Unidas, $\mathrm{N}^{\mathrm{o}}$ de venta: S.86.XVII.12.

Oman, C. (2000), Policy Competition for Foreign Direct Investment: A Study of Competition among Governments to Attract FDI, París, Organización de Cooperación y Desarrollo Económicos (OCDE).

Rugman, A. y R. Hodgetts (2001), "The end of global strategy", European Management Journal, vol. 19, № 4, Amsterdam, Elsevier.

Rugman, A.M. y S. Collinson (2004), "The regional nature of the world's automotive sector", European Management Journal, vol. 22, No 5 , Amsterdam, Elsevier.

Schlie, E. y G. Yip (2000), "Regional follows global: strategy mixes in the world automotive industry", European Management Journal, vol. 18, $\mathrm{N}^{\mathrm{o}}$ 4, Amsterdam, Elsevier.

Soloaga, I. y A.L. Winters (2001), "Regionalism in the nineties: what effect on Trade?", The North American Journal of Economics and Finance, vol. 12, $\mathrm{N}^{\circ} 1$, Amsterdam, Elsevier. 